Vol. 13, No 1-2 | 1er semestre 2019 - 2ème semestre 2019 Les industries culturelles à la conquête des plateformes?

\title{
Quelle « plateformisation » de l'information? Collusion socioéconomique et dilution éditoriale entre les entreprises médiatiques et les infomédiaires de l'Internet
}

\section{Franck REBILLARD et Nikos SMYRNAIOS}

\section{(2) OpenEdition \\ Journals}

\section{Édition électronique}

URL : http://journals.openedition.org/ticetsociete/4080

DOI : $10.4000 /$ ticetsociete. 4080

Éditeur

Association ARTIC

Édition imprimée

Pagination : 247-293

Référence électronique

Franck REBILLARD et Nikos SMYRNAIOS, «Quelle « plateformisation » de l'information ? Collusion socioéconomique et dilution éditoriale entre les entreprises médiatiques et les infomédiaires de I'Internet », tic\&société [En ligne], Vol. 13, № 1-2 | 1er semestre 2019 - 2ème semestre 2019, mis en ligne le 20 avril 2019, consulté le 27 mai 2019. URL : http://journals.openedition.org/ticetsociete/4080 ; DOI : 10.4000/ticetsociete.4080 
tic\&société - 13(1-2), 2019

\title{
Quelle « plateformisation » de l'information? Collusion socioéconomique et dilution éditoriale entre les entreprises médiatiques et les infomédiaires de l'Internet
}

\begin{abstract}
Franck Rebillard
Franck Rebillard est professeur en sciences de l'information et de la communication à l'université Sorbonne Nouvelle - Paris 3 , où il dirige l'unité de recherche Irméccen (Institut de recherche Médias, cultures, communication et numérique). Ses travaux portent principalement sur le journalisme en ligne, objet qu'il approche en croisant socioéconomie et analyse de discours. Auteur de nombreux articles à ce sujet, publiés dans l'espace scientifique francophone comme anglophone, il est également l'auteur d'ouvrages plus généraux portant sur l'Internet " participatif » (2007), le pluralisme de l'information (2013), la transition numérique des industries culturelles et médiatiques (2016). franck.rebillard@sorbonne-nouvelle.fr
\end{abstract}

\section{Nikos Smyrnaios}

Nikos Smyrnaios est maître de conférences en sciences de l'information et de la communication à l'université de Toulouse, membre du Lerass (Laboratoire d'études et de recherches appliquées en sciences sociales). Ses sujets de recherche concernent l'économie politique des médias en ligne, le journalisme numérique et les usages politiques des réseaux sociaux numériques. Son ouvrage Les Gafam contre l'internetUne économie politique du numérique a été publié en France en 2017 et traduit en anglais en 2018. nicolaos.smyrnaios@iuttlse3.fr 
Quelle « plateformisation » de l'information ? Collusion socioéconomique et dilution éditoriale entre les entreprises médiatiques et les infomédiaires de l'internet

\title{
Quelle « plateformisation » de l'information? Collusion socioéconomique et dilution éditoriale entre les entreprises médiatiques et les infomédiaires de l'internet
}

\begin{abstract}
Résumé : La production et la diffusion de l'information en ligne sont actuellement caractérisées par un basculement de la consommation vers les terminaux mobiles et par une circulation privilégiée via les réseaux sociaux numériques. Sur le plan théorique et historique, nous proposons d'analyser ces phénomènes, fréquemment qualifiés de "plateformisation ", comme une accentuation du processus d'infomédiation, en conciliant économie politique de la communication, analyse de discours médiatique et technosémiotique de l'Internet. Comparant des matériaux empiriques accumulés dans le passé à quelques indicateurs plus récents émanant d'entretiens avec des acteurs de la filière et d'une analyse des interfaces de consultation d'information sur supports mobiles, nous appuierons l'hypothèse générale d'une évolution à la fois plus intégrative et plus asymétrique des relations entre infomédiaires de l'Internet et entreprises médiatiques, pouvant se dénommer collution (collusion socioéconomique et dilution éditoriale).
\end{abstract}

Mots-clés : infomédiation, plateformes, journalisme, information en ligne, économie politique.

Abstract: The production and distribution of news is currently characterized by both a switchover to the consumption of online information on mobile terminals, and a predilection for its circulation through social media. We propose a historical and theoretical analysis of this phenomenon of "platformization" as an accentuation of the infomediation process. Our analysis strives to reconcile the political economy of communication, media discourse analysis and technosemiotics of the Internet. Comparing empirical material from the past with some more recent indicators identified from interviews with industry players and an analysis of mobile news interfaces, we support the general hypothesis of an evolution towards more integrated and more asymmetrical relations between infomediaries and media 
companies that can be termed collution (socioeconomical collusion and editorial dilution).

Keywords: infomediation, platforms, journalism, online news, political economy.

Resumen: La producción y difusión de información sobre Internet se caracteriza por un cambio en el consumo mediante la utilización de terminales móviles y por una utilización de las redes sociales digitales. En el nivel teórico e histórico, se propone analizar estos fenómenos, descritos como "plataformización", como una acentuación del proceso de infomediación, reconciliando la economía política de la comunicación con el análisis del discurso de los medios y con la tecno-semiótica de Internet. A partir de la comparación de los materiales empíricos acumulados en el pasado y de algunos indicadores más recientes que surgen de entrevistas con actores de la industria, así como de un análisis de las interfaces de consulta de información móvil, la hipótesis general que se plantea es la existencia de una evolución a la vez más integradora y más asimétrica entre los infomediarios de Internet y las empresas de medios de comunicación; esto hace que puede hablarse de colución (colusión socioeconómica y dilución editorial).

Palabres claves: infomediación, platforms, periodismo, información sobre Internet, economía política. 
Quelle « plateformisation » de l'information ? Collusion socioéconomique et dilution éditoriale entre les entreprises médiatiques et les infomédiaires de l'internet

\section{Introduction}

Le milieu des années 2010 aura été marqué par deux phénomènes majeurs pour l'information en ligne. D'une part, la consultation de l'information s'est déplacée en direction des terminaux mobiles. Aux États-Unis, où les tendances en la matière sont souvent annonciatrices de ce qui va se passer dans d'autres pays industrialisés, une étude comparative portant sur un panel des principaux médias montre que la consultation de l'actualité sur terminaux mobiles (téléphones et tablettes) est devenue supérieure à celle sur ordinateur en 2015 (Pew Research Center [Pew], 2016). Un mouvement semblable a été enclenché dans d'autres pays, comme en témoigne une étude analogue portant sur un panel des principaux médias français : de 2014 à 2015, la part des visites effectuées depuis un smartphone est passée de $31 \%$ à $38 \%$ (AT-Internet, 2015). Début 2018, plus de la moitié de la population de ces deux pays (51\% en France et $56 \%$ aux États-Unis) utilisait le téléphone mobile pour s'informer sur l'actualité (RISJ, 2018).

Ce déplacement de la consultation vers les terminaux mobiles a d'autre part accentué le croisement des pratiques d'information avec des pratiques de communication (conversation, échange de photographies et vidéos...) qu'ont pu exploiter les opérateurs de réseaux sociaux numériques (RSN). Le recours aux RSN pour s'informer sur l'actualité a connu une croissance forte et continue au milieu de la présente décennie, avant de se tasser quelque peu ces dernières années (RISJ, 2018). Cette pratique a toutefois atteint un niveau élevé, pouvant concerner jusqu'à $68 \%$ de la population aux ÉtatsUnis en 2018 (Pew, 2018a) et $45 \%$ de la population française en 2017 (Pew, 2018b).

Ces deux phénomènes - migration vers les terminaux mobiles et emploi des RSN pour s'informer - sont concomitants du lancement de services de RSN dédiés à la consultation sur mobile en 2015: Snapchat Discover en janvier, Facebook Instant Articles en mai. Cette même année 2015, d'autres 
firmes comptant au rang de l'oligopole des GAFAM ${ }^{1}$ ont suivi le mouvement, avec Apple News en mai et le procédé AMP (Accelerated Mobile Pages) de Google en octobre (pour une chronologie complète et détaillée, cf. Bell et Owen, 2017).

\section{I.1 L'infomédiation, phénomène peu étudié}

Une telle incursion dans la filière de l'information de la part de firmes issues des secteurs des technologies numériques et de l'informatique ne date évidemment pas du milieu de la présente décennie. Elle existe en fait depuis les débuts d'Internet "grand public », au début des années 1990, autrement dit depuis la sortie d'Internet des seuls univers militaires et académiques au profit d'usages plus diversifiés et commerciaux. Rappelons par exemple que l'un des premiers magazines en ligne, Slate, fut créé en 1996 par Microsoft ${ }^{2}$.

Toutefois, c'est surtout à partir du début des années 2000 que ces firmes ont commencé à peser de tout leur poids sur la filière de l'information en ligne, en se plaçant comme intermédiaires dans l'accès à l'information. À cet égard, une initiative majeure fut la création de Google News en 2002 (2003 pour sa version française), imaginé comme une réponse à la cacophonie médiatique constatée au moment des attentats du 11 septembre 2001. Ce service emblématique illustrait de façon plus générale la capacité de l'informatique à gérer la multitude des flux d'information en ligne et à organiser leur consultation individualisée par les internautes, autrement dit à assurer une activité d'infomédiation (Smyrnaios, 2015).

Cette activité d'infomédiation, initiée par de puissants moteurs de recherche comme Google, mais aussi par des services de veille comme la start-up française Net2One créée à la fin des années 1990, s'est ensuite développée tout au long des quinze dernières années jusque vers les RSN et les applications pour terminaux mobiles, au point que l'on puisse distinguer infomédiation algorithmique, infomédiation sociale et infomédiation applicationnelle ou transactionnelle (Rebillard et Smyrnaios, 2011). Cette place croissante prise par

\footnotetext{
${ }^{1}$ L'acronyme Gafam regroupe les entreprises Google, Amazon, Facebook, Apple, Microsoft.

${ }^{2}$ Racheté ensuite par la Washington Post Company, Slate a aussi connu une version française à partir de 2009.
} 
Quelle « plateformisation » de l'information ? Collusion socioéconomique et dilution éditoriale entre les entreprises médiatiques et les infomédiaires de l'internet

l'infomédiation dans la filière de l'information en ligne a été l'objet de relativement peu de travaux pendant plusieurs années, à l'exception des ceux précédemment cités et de quelques études et réflexions plus ciblées (par ex. : del ÁguilaObra, Padilla-Meléndez et Serarols-Tarrés, 2007; Siapera, 2013). Ce n'est que tout récemment, en écho à des incitations à réorienter les recherches internationales sur le journalisme et les médias au-delà des frontières des seules entreprises productrices d'information (news organisations), vers une dimension sociotechnique plus marquée (Lewis and Westlund, 2015) et notamment vers une prise en compte du poids de l'informatique et des programmes algorithmiques (Napoli, 2014), que des recherches empiriques se sont multipliées à ce sujet (Just et Latzer, 2017; Nechuchstai, 2018; Nielsen et Ganter, 2018; Tambini et Labo, 2016; Tandoc et Maitra, 2018).

\section{I.2 Un engouement pour la plateformisation en question}

Cette insistance soudaine, dans la littérature scientifique internationale (anglophone), sur "l'importance de ne pas restreindre l'étude des changements dans le domaine de la production d'information aux seuls journalistes, mais de s'intéresser aussi aux autres acteurs tels que les managers, les informaticiens, les publics et les entités extérieures comme les opérateurs de plateformes ${ }^{3}$ " (Kalogeropoulos et Nielsen, 2017, p. 16), semble étroitement liée aux deux phénomènes conjoncturels mis en exergue au début de ce texte. De façon emblématique, la recherche citée précédemment s'attache par exemple à montrer que " les vidéos d'information en ligne sont pour la plupart produites d'abord et avant tout (1) pour être vues sur des smartphones, plutôt que sur des microordinateurs, et (2) pour être diffusées sur des plateformes comme Facebook et YouTube, plutôt que sur les sites web des médias qui les ont produites ${ }^{4} »($ p. 4).

De façon plus générale, ces travaux récents documentent de

\footnotetext{
${ }^{3}$ Traduction libre de : " the importance of studying not only journalists, but also other actors including managers, technologists, audiences, and outside entities like platform companies when researching changes in news production $"$.

${ }^{4}$ Traduction libre de : " most online video news is produced first and foremost (1) to be viewed on smartphones rather than on personal computers and (2) distributed via platforms like Facebook and YouTube rather than on the websites of news organizations themselves ".
} 
façon utile la situation contemporaine, soulignant l'emprise des opérateurs de plateformes sur les entreprises productrices d'information. Les analyses proposées à cet égard ne nous semblent toutefois pas complètement satisfaisantes, principalement parce qu'elles reposent sur l'impression présentiste - de l'irruption d'un nouvel acteur à l'activité souvent mal définie: la plateforme. Nous proposons par conséquent de les compléter sur le plan théorique et historique.

Nous plaiderons ainsi pour une réinscription conceptuelle de la plateformisation vis-à-vis de l'activité d'infomédiation. Pour cela, nous nous efforcerons notamment de concilier économie politique de la communication, analyse de discours médiatique et technosémiotique de l'Internet, afin de considérer conjointement les parentés socioéconomiques et sémiodiscursives de ces phénomènes. Observant les relations entre infomédiaires et entreprises médiatiques depuis plusieurs années, nous proposerons alors d'analyser la plateformisation avant tout comme une accentuation de l'infomédiation. Comparant des matériaux empiriques accumulés dans le passé avec quelques indicateurs plus récents résultant d'entretiens avec des acteurs de la filière et d'une analyse des interfaces de consultation de l'information sur supports mobiles, nous appuierons l'hypothèse générale d'une évolution à la fois plus intégrative et plus asymétrique des relations entre infomédiaires de l'Internet et entreprises médiatiques, sur le plan socioéconomique comme sémiodiscursif.

\section{Développements théoriques}

Dans cet article, nous nous intéressons à l'évolution de la production et de la diffusion de l'information d'actualité. Parce que celle-ci n'est pas un bien tout à fait comme les autres, en particulier parce qu'elle joue un rôle de premier plan dans la vie démocratique, il paraît pertinent de ne pas lui appliquer une analyse économique stricto sensu. L'économie politique lui sera ainsi préférée, considérant que la production et la diffusion de l'information d'actualité dans la société doivent être analysées au regard de problématiques politiques relevant de l'intérêt général, telles que le financement d'une pluralité de médias ou la capacité d'expression d'une multitude d'opinions. Pour cette même raison, il paraît aussi nécessaire de s'intéresser de près 
Quelle « plateformisation » de l'information ? Collusion socioéconomique et dilution éditoriale entre les entreprises médiatiques et les infomédiaires de l'internet

aux discours et aux formes médiatiques ainsi mis en circulation dans l'espace public. Cela suppose de revisiter en partie l'économie politique de la communication pour y rehausser la strate d'analyse de la nature des biens produits, en l'occurrence la nature des informations ensuite mises en ligne.

\section{1 Économie politique et information en ligne}

Une telle préoccupation pour les "contenus » est en réalité assez ancienne en économie politique de la communication. Elle est même une des fondations de la théorie des industries culturelles, également dénommée the cultural industries approach à l'international (Hesmondhalgh, 2013), un des courants de l'économie politique de la communication prenant pour objet l'information, mais aussi la culture ${ }^{5}$.

Rappelons que, au sein de ce courant, contrairement à une théorisation monolithique de l'industrie culturelle, les industries culturelles et médiatiques sont volontairement pensées au pluriel. On s'attache à y décrire les différentes modalités de production et de diffusion au sein de chaque filière: livre, musique enregistrée, cinéma, presse écrite, radio, télévision. Et, de fait, une analyse comparative de ce type, menée depuis plusieurs décennies déjà, a permis de déceler à la fois un certain nombre de points communs dans l'organisation de ces filières (à commencer par la nécessité, pour les acteurs placés au centre des filières, de concilier la création intellectuelle en amont avec des impératifs commerciaux en aval) et des variations tenant notamment aux modes de financement des différents biens produits et diffusés.

\subsubsection{Modèles socioéconomiques et nature des informations}

Nous souhaiterions ici insister sur ce dernier point, souvent oublié ou minoré : les caractéristiques sociales et économiques identifiées au sein d'une filière sont liées au type de biens qui y est produit. La différenciation initiale entre modèle éditorial et modèle de flot (Miège, Pajon et Salaün, 1986) recouvre l'écart existant entre, d'un côté, des biens susceptibles d'être acquis

\footnotetext{
${ }^{5}$ La production et la diffusion de la culture, tout comme celles de l'information, posent à la société des questions là encore essentielles, comme celles de la créativité artistique ou de la conservation du patrimoine.
} 
parce que pérennes (leur valeur d'usage ne s'épuise pas après une première consommation) et, de l'autre côté, des biens éphémères et difficilement appropriables. À cette distinction, on pourrait ajouter celle entre biens rivaux, comme les livres, et non rivaux, comme la transmission hertzienne des programmes audiovisuels, qui facilite l'acquisition individuelle pour les premiers et accentue le besoin d'un financement indirect pour les seconds. C'est bien parce que le modèle éditorial correspond à des filières (livre, musique enregistrée) de production et de diffusion de biens pérennes et appropriables qu'on y fait appel à un financement direct par les lecteurs et mélomanes et que les remontées de recettes engendrées se distribuent entre l'éditeur/producteur au centre et des créateurs œuvrant en mode projet en amont. Inversement, le modèle de flot correspond à d'autres filières (radio, télévision) de production et de diffusion de biens éphémères et difficilement appropriables par les téléspectateurs et les auditeurs, des biens devant être créés en continu, financés indirectement par les annonceurs (et les pouvoirs publics) et plaçant le programmateur (de " grille », de chaîne) au centre.

En tant que modèles idéal-typiques au sens weberien, ces deux modèles génériques ont permis, au fil du temps, de rendre compte de l'agencement de plusieurs autres filières (voire d'alimenter les débats théoriques à propos des industries créatives, cf. Bouquillion, Miège et Mœglin, 2013), à commencer par celle de la presse et de l'information qui nous concerne plus particulièrement ici. Pendant plusieurs années, la filière de la presse écrite a ainsi été appréhendée par la théorie des industries culturelles comme un modèle intermédiaire entre éditorial et flot, sur des critères essentiellement économiques puisqu'elle tirait son financement du double marché des lecteurs et des annonceurs. Or ce financement à la fois direct et indirect était lié à des contenus eux-mêmes hybrides entre biens pérennes (par ex., dossier de presse magazine) et biens éphémères (par ex., brève dans un quotidien), ce qui a été beaucoup moins souligné. Pourtant, la prise en compte de la nature des informations se révèle très utile pour analyser les évolutions ultérieures de la presse et renforcer la validité du raisonnement en matière de modèles médiatiques (Rebillard, sous presse) ; ainsi, au début des années 2000 , la balance a plutôt penché du côté du modèle de flot, avec le lancement des quotidiens gratuits (information très factuelle, donc éphémère, 
Quelle « plateformisation » de l'information ? Collusion socioéconomique et dilution éditoriale entre les entreprises médiatiques et les infomédiaires de l'internet

et financée uniquement par les annonceurs), puis s'est inversée autour de 2010 avec le succès de la formule du "mook » (magazine-book proposant une information pérenne, sous un format proche du livre, sans publicité et vendu en librairie).

\subsubsection{Modèle(s) de l'information en ligne}

Les diversifications touchant la presse écrite peuvent ainsi être interprétées à la lumière des modèles issus de la théorie des industries culturelles, avec un surcroît d'intelligibilité procuré par la prise en compte des modalités sémiodiscursives au-delà des seules modalités socioéconomiques. Procédant de la sorte, nous avons ainsi pu avancer, à partir d'une analyse quantitative de discours de sites web d'information générale et politique montrant un fort dualisme éditorial entre concentration sur un nombre réduit de sujets portés à la une de l'agenda numérique et dispersion en de nombreux autres sujets abordés plus isolément, que la filière de l'information en ligne pouvait s'inscrire dans plusieurs modèles (Rebillard, 2012). Au début des années 2010, les sites issus de médias traditionnels (sites de journaux et magazines, de stations de radio, de chaînes de télévision) reproduisaient assez naturellement, en ligne, le modèle éditorial et le modèle de flot. Mais, plus globalement, la filière de l'information en ligne semblait être régie par un autre modèle, celui du courtage informationnel, modèle hérité de l'information spécialisée et renforcé par la généralisation de l'infomédiation sur l'Internet (Mœglin, 2007).

Le développement de l'information en ligne s'inscrit de fait dans une évolution plus large de rapprochement du secteur des industries culturelles et médiatiques avec ceux des télécommunications et de l'informatique. Les filières prises dans ce « tournant numérique » le sont à des degrés divers, mais, là encore, un certain nombre de phénomènes transversaux apparaissent. Parmi eux, l'un des plus notables a été l'arrivée de nouveaux acteurs, dont la place intermédiaire entre production et distribution a été très vite perçue comme centrale dans les filières en cours d'agencement: "la soi-disant "désintermédiation" générale des marchés masque de nouvelles formes de "ré-intermédiation" par de nouveaux acteurs qui ont émergé de l'environnement numérique. [Ceuxci] concentrent et orientent le trafic, mais assurent aussi de 
nouvelles fonctions de packaging et de commercialisation ${ }^{6}$ » (Bustamante, 2004, p. 808).

La réintermédiation dont il est question ici est l'infomédiation. Et comme Bustamante l'indique, l'infomédiation comporte des composantes à la fois sociale (guidage des internautes), économique (commercialisation, notamment publicitaire), et éditoriale (packaging ou mise en forme des contenus). L'alliage entre ces différentes composantes soutient le modèle du courtage informationnel. On en trouve une déclinaison dans la filière de l'information en ligne où, par exemple, la mise en relation - outillée par l'informatique - entre les internautes (composante sociale) et des biens archivables, modulables et pointables hypertextuellement (composante éditoriale) se double de liens commandités par des annonceurs et individualisés (composante économique). Nous proposons d'examiner plus en détail cette structuration de la filière de l'information en ligne par l'infomédiation pour mieux voir si la plateformisation constitue à ce titre une rupture ou une continuité.

\subsection{Infomédiation et plateformisation}

Appréhender convenablement ce qui est décrit aujourd'hui sous les traits de la plateformisation requiert selon nous de resituer ces derniers soubresauts dans une histoire plus longue de l'infomédiation.

L'infomédiation a été pensée dès les années 1970 comme un recours à la médiation des machines pour gérer une surcharge d'information grandissante avec les réseaux informatisés, dépassant les capacités de l'esprit humain. Au cours des années 1980, elle a connu une première forme de concrétisation dans des systèmes d'interrogation de bases de données accessibles à distance (pour une généalogie plus complète de la notion, voir Goyette-Côté, dans Rebillard et al., 2011). Depuis, l'infomédiation consiste plus généralement en une activité d'appariement, outillée par l'informatique, entre des contenus produits par des tiers et des demandes

6 Traduction libre de : " the self-proclaimed general "disintermediation" of markets hides new forms of "re-intermediation", with new agents that have emerged from the digital environment. [These ones] concentrate and orientate the mass traffic, but also assume new functions of packaging and commercialization. " 
Quelle « plateformisation » de l'information ? Collusion socioéconomique et dilution éditoriale entre les entreprises médiatiques et les infomédiaires de l'internet

individualisées, incluant la prise en charge des modalités techniques de circulation en ligne et d'assemblage éditorial des éléments concernés.

À ce titre, l'infomédiation devient l'activité centrale dans les filières se développant sur l'Internet (information en ligne, musique en ligne, etc.), car celles-ci présentent deux caractéristiques qui les différencient des filières précédentes des industries culturelles reposant sur l'imprimé (livre, presse) et sur l'audiovisuel (télévision, cinéma, radio, musique enregistrée). En amont des filières, l'offre s'accroît considérablement : aux côtés des contenus professionnels du moment sont rendus disponibles les contenus archivés au cours du temps, et s'y ajoute une multitude de contenus amateurs. En aval des filières, la demande s'exerce de manière différente, selon une logique de l'accès qui la rend beaucoup plus personnalisable. Dans une telle configuration, l'infomédiation se positionne comme nouvelle intermédiation au sein de la filière : d'une part, elle dépasse l'édition en tenant davantage compte des préférences d'internautes désormais moins soumis aux choix éditoriaux ou aux grilles de programmation imposés "d'en haut»; d'autre part elle ne se réduit pas à la distribution puisqu'opérant un réagencement des contenus en exploitant de grandes quantités de données collectées aussi bien du côté de l'offre (fragments de textes ou d'images, métadonnées et descripteurs informatiques) que de la demande (éléments de profil sociodémographique, traces d'usage).

\subsubsection{L'infomédiation de l'information en ligne}

L'infomédiation a ainsi rapidement pris une position centrale dans la filière de l'information en ligne, dans le contexte plus global de mise en place d'une économie oligopolistique dominée par les GAFAM (Smyrnaios, 2017). On peut l'analyser à partir du cadre théorique précédemment dessiné, à savoir celui d'une économie politique de la communication attentive à la nature des biens produits et diffusés. Dans cette optique, les différentes dimensions - socioéconomiques et sémiodiscursives - du modèle du courtage informationnel, appliqué à la filière de l'information en ligne, seraient les suivantes :

- Nature du bien: contrairement au journal écrit, 
Franck Rebillard et Nikos Smyrnaios

radiophonique ou télévisé qui forme un bien-système produit par les entreprises médiatiques, l'information offerte par l'infomédiation est atomisée et modularisée sous la forme de l'article, de l'illustration photographique ou infographique, de l'extrait sonore ou vidéo. Ces modules peuvent être constamment réagencés par les infomédiaires pour alors constituer un nouveau type de biens-systèmes sous la forme de services en ligne d'actualité.

- Mode de financement: la publicité est dominante, mais il s'agit d'une promotion personnalisée, adaptée aux préférences des internautes gérées lors de l'activité d'infomédiation. Secondairement, il peut être fait appel à une rémunération par l'utilisateur final, notamment sous la forme de micropaiements de l'information sur lesquels des commissions peuvent être prélevées par les infomédiaires.

- Modalités de production: alors qu'un magistère de l'offre a longtemps gouverné les façons de travailler des journalistes, ces derniers ont tendance à davantage prendre en compte la demande, notamment par des outils et des métriques qui le plus souvent appartiennent aux infomédiaires eux-mêmes, permettant de connaître les tendances de consultation article par article (ou module par module).

Ainsi, une forme de cohérence apparaît entre modularisation-linkage-réassemblage de l'information, courtage promotionnel et production sur mesure. Chacune de ces dimensions peut connaître quelques variations selon que l'infomédiation repose essentiellement sur une programmation algorithmique (par ex., Google limite souvent la modularisation de l'information aux titres et à quelques lignes de description des articles qu'il recompose continuellement à partir d'une multitude de critères statistiques), intègre les relations sociales entre internautes (par ex., Facebook intègre les actions de recommandation - like, comment, share - de ses membres), ou est organisée à partir d'applications informatiques ad hoc (par ex., Apple ajoute à la publicité personnalisée des possibilités de financement par le micropaiement). Cependant, l'alliage de ces dimensions sémiodiscursives et socioéconomiques demeure globalement, et confère aux infomédiaires une place centrale dans la filière de l'information en ligne, au détriment des 
Quelle « plateformisation » de l'information ? Collusion socioéconomique et dilution éditoriale entre les entreprises médiatiques et les infomédiaires de l'internet

producteurs d'information (Rebillard et Smyrnaios, 2011).

\subsubsection{Spécificités potentielles de la plateformisation}

La question que nous posons dans cet article est de savoir si l'agencement de la filière de l'information en ligne autour de l'infomédiation a notablement évolué ces derniers temps, en particulier avec l'avènement annoncé des plateformes.

Depuis quelques années, plusieurs travaux et réflexions ont été conduits à ce sujet, au point de voir émerger un ensemble se dénommant platform studies. Cet ensemble, même s'il ne revendique pas de filiations directes avec l'économie politique de la communication, présente toutefois de nombreuses parentés avec celle-ci (Winseck, 2016). On retrouve ainsi, dans ce type d'analyse, un intérêt à étudier les dimensions à la fois sociales, économiques et éditoriales des phénomènes communicationnels, avec toutefois une attention plus forte visà-vis des dimensions proprement techniques, point qui distinguerait les platform studies (Plantin et al., 2018). De fait, dans le cas de l'information en ligne, les analyses que nous avons réalisées à partir du modèle du courtage informationnel semblent assez voisines de celles présentées tout récemment sous l'appellation de "plateformisation de l'information " (platformization of news) : "La plateformisation [...] marque le passage, pour la production et la diffusion d'information, d'un processus de choix éditoriaux à un processus guidé par la demande au sein duquel le contenu est continuellement modulé et réassemblé sur la base de données collectées à propos des utilisateurs et de leurs pratiques ${ }^{7}$ "(Poell et al., 2017, p. 10).

Les caractéristiques relevées par les platform studies à propos de l'information en ligne recoupent ainsi en grande partie les éléments que nous avions identifiés quelques années plus tôt dans nos travaux et nos réflexions sur l'infomédiation, à savoir le passage à une logique de l'accès individualisé nécessitant le recours à des procédés informatiques pour sélectionner des contenus modularisables. Ceci nous incite à penser que les deux termes d'infomédiation et de plateformisation recouvrent en fait un même phénomène, abordé de façons légèrement différentes. L'économie politique

\footnotetext{
${ }^{7}$ Traduction libre de : « Platformization i[...] entails a shift form an editorially driven to a demand-driven nexs production and distribution process, in which content is continuously modulated, and repackaged, informed by datafied user feedback ».
} 
de la communication tout comme les platform studies permettent de s'intéresser aux dimensions sociales, économiques et éditoriales, avec toutefois une antériorité pour la première, qui autorise l'historicisation du phénomène, et un tropisme sociotechnique pour la seconde, qui permet d'en prendre la pleine mesure.

À cet égard, un travail issu des platform studies se distingue (Helmond, 2015). Outre le fait d'embrasser pleinement la dimension sociotechnique de l'Internet et en particulier sa " programmabilité », il possède une véritable dimension diachronique pour décrire la «double logique de la plateformisation ", faite de "décentralisation des éléments des plateformes dans le web » (par ex. bouton like, vidéo YouTube incrustés sur des sites tiers) et, en retour, de « recentralisation des données rendues traitables par les plateformes ${ }^{8}$ " (Helmond, 2015, p. 1). Ce double mouvement, entamé au milieu des années 2000, a été annoncé en même temps que le passage à un Web 2.0, mais est passé plus inaperçu que les promesses du Web participatif. Pourtant, il se concrétise et s'intensifie à mesure que les principaux acteurs de l'Internet accroissent leur emprise : cette emprise passe à la fois par une ouverture vers l'extérieur, en permettant aux autres acteurs de développer des contenus et des applications compatibles, notamment par les API, et par un rapatriement des données ainsi générées au sein de la plateforme.

La perspective de ce travail, diachronique, est congruente avec notre hypothèse générale d'une accentuation de l'infomédiation qu'illustre la plateformisation. Nous la retenons d'autant plus qu'elle ajoute une dimension sociotechnique (valeur ajoutée scientifique des platform studies) susceptible d'enrichir l'approche de l'économie politique de la communication au moment d'appréhender le tournant numérique, et nous l'appliquons donc à l'étude de l'information en ligne.

\footnotetext{
8 Traduction libre de : « dual logic of platformization » « decentralization of platform features into the web » et « recentralization of platform ready data ».
} 
Quelle « plateformisation » de l'information ? Collusion socioéconomique et dilution éditoriale entre les entreprises médiatiques et les infomédiaires de l'internet

\section{Indices empiriques}

Partant de l'idée que la double logique de plateformisation constituerait un processus d'intensification de l'infomédiation dans la filière de l'information en ligne, nous tenterons ainsi d'examiner dans quelle mesure ce processus se décline au niveau de chacune des dimensions constitutives du modèle du courtage informationnel. Ceci nous amène d'une part à prendre en considération aussi bien les dimensions socioéconomiques que les dimensions sémiodiscursives, tout en ajoutant une pointe sociotechnique désormais plus affirmée. Cela nous conduit d'autre part à déceler, dans le cas de l'information en ligne en France, des indices empiriques qui s'inscriraient dans les tendances suivantes, déjà identifiées par la littérature internationale sur la plateformisation :

- Mode de financement: les circuits habituels de remontée de recettes de la filière de l'information en ligne se doublent de data channels (Helmond, 2015) où les données sur les internautes sont recentralisées par les infomédiaires, ce qui accentue leur pouvoir sur le marché de la publicité personnalisée. Cette tentative de captation des données utilisateurs par les intermédiaires techniques qui a commencé à la fin des années 1990 serait alors totalement aboutie.

- Modalités de production : en plus d'avoir à disposition des outils permettant de cibler et de calibrer leurs publications pour optimiser leur audience, les journalistes sont désormais formés et conseillés directement par les infomédiaires (Nechushtai, 2018), au point de quasi-intégrer ces entreprises comme des sous-traitants.

- Nature des biens: l'information n'est pas seulement modularisée au point de devenir une "contingent commodity" (Poell et al., 2017) ajustable aux différents formats de diffusion des infomédiaires, elle en vient désormais à être hébergée directement dans le périmètre de la plateforme.

Les deux premiers points seront renseignés sur la base d'entretiens menés auprès d'acteurs français de la filière de l'information en ligne, et le troisième point sera complété par une analyse technosémiotique des interfaces de consultation de l'information sur téléphone mobile (smartphone). Nous 
souhaitons souligner que les éléments empiriques livrés ici ont un simple statut d'indices. D'une part, dans le cadre formel imposé par cet article, il n'est pas possible de présenter une analyse empirique vraiment substantielle si l'on veut aborder l'ensemble des dimensions (socioéconomique, sémiodiscursive, sociotechnique) que requiert notre cadre théorique ; par conséquent, nous ne livrerons ici que des bribes empiriques qui méritent d'être complétées par d'autres travaux. Au-delà de cet aspect quantitatif réduit de notre travail empirique qui amène à parler d'indices, nous employons d'autre part ce terme pour souligner le statut très situé de la période sur laquelle nous nous penchons : celle-ci est limitée dans le temps au regard de l'évolution de l'infomédiation et, là encore, il faudra renouveler ce type d'enquête et d'analyse de corpus dans les années à venir pour voir si l'accentuation jusqu'à la plateformisation se confirme ou non.

\subsection{Collusion socioéconomique}

Depuis quinze années maintenant, au travers de travaux individuels ou de projets collectifs dirigés par nos soins, près de cinquante entretiens ont été effectués auprès d'acteurs de la filière de l'information en ligne en France, se répartissant entre 31 du côté des entreprises médiatiques et 18 du côté des infomédiaires. Comme indiqué précédemment, pour des contraintes de format, nous ne mobiliserons ici qu'une partie réduite de ces entretiens, prioritairement ceux menés lors d'une dernière vague, fin $2016^{9}$. Cependant, nous interprèterons leur teneur à l'aune des entretiens menés depuis le début des années $2000^{10}$ (entretiens cités avec davantage de parcimonie pour les mêmes raisons de taille limitée de cet article), afin de mieux saisir les nouveautés potentielles se rattachant à la plateformisation.

\footnotetext{
${ }^{9}$ S'inscrivant dans le projet de recherche Enjeux socioéconomiques de la diffusion d'informations d'actualité sur l'internet, projet financé par le ministère de la Culture et de la Communication, ces entretiens ont été conduits par Andrea Wagemans, que les auteurs tiennent à remercier.

${ }^{10}$ Un retour rétrospectif plus complet sur les entretiens menés lors de ces quinze années (entretiens effectués par les auteurs et Marc-Olivier Goyette-Côté, qu'ils remercient également), est présenté dans un autre texte, rédigé en langue anglaise (Smyrnaios et Rebillard, article en cours d'évaluation), texte qui prolonge le présent article sur son versant socioéconomique.
} 
Quelle « plateformisation » de l'information ? Collusion socioéconomique et dilution éditoriale entre les entreprises médiatiques et les infomédiaires de l'internet

\subsubsection{Relations interindividuelles}

Lorsque Google a lancé son service dédié à l'actualité Google News au début des années 2000 en France, cela s'est fait depuis la Californie, sans en avoir aucunement prévenu les éditeurs de presse français :

«Quand j'ai vu Google News sortir aux États-Unis, j'ai demandé qu'on l'implémente rapidement en France. Pour moi, c'était une évidence qu'ils allaient demander aux éditeurs leur permission de pouvoir être cités dans le service, mais ils ne l'ont jamais fait! Une fois que le truc a été créé en français et que j'ai réalisé qu'ils n'ont rien demandé aux éditeurs, j'ai été sidéré. [...] Ce n'est pas que je n'ai pas fait mon boulot, c'est que ce n'était pas mon boulot. [...] Pour faire simple, je n'avais moi, en tant que country manager, absolument aucune latitude de gérer Google Actualités. Dans Google, l'organisation n'est pas pyramidale, c'est-à-dire vous n'avez pas un chef, un vrai DG par pays qui gère tout, le marketing, les relations presse, les équipes commerciales et les supports. C'est moi qui les recrutais, mais, une fois qu'ils étaient dans la société, je n'étais plus leur patron : c'est ce qu'on appelle une organisation matricielle. " (Franck Poisson, ancien directeur général, Google France, 12 juillet 2005)

Les relations entre les deux parties ont par la suite été très tendues, émaillées de menaces de désindexation du moteur ou encore d'attaques en justice. Les pourparlers ont principalement eu lieu entre le principal infomédiaire de l'époque, Google, et le Geste (groupement d'éditeurs de contenus numériques rassemblant les principaux médias nationaux français), ou certains de ses membres parmi les plus influents :

" Ça fait des années qu'on a des dialogues, moi ça fait six ans que je suis chez Google et, dès ma première année, j'avais des rencontres avec le Geste et avec des membres individuels [...]. [I] y a souvent eu cette confusion en disant Google s'approprie l'information et donc devrait rémunérer des éditeurs à ce titre-là. Nous on dit non, si on intègre vraiment vos articles sur notre site et qu'on garde les lecteurs chez nous, là ça serait logique, l'échange de valeur, il ne serait pas fair. Dans la mesure où l'on 
continue de permettre aux gens de vous trouver et d'aller consulter l'article et après on estime qu'on a un dosage, on leur donne suffisamment d'informations pour qu'ils déterminent ce qui est suffisamment pertinent pour leur permettre de faire un choix éclairé. " (Yoram Elkaïm, Head of Legal, Google France, 19 juillet 2011)

Dix ans plus tard, à côté d'accords bilatéraux et ponctuels pouvant d'ailleurs donner lieu à versement monétaire - achat par Google à l'AFP d'une " licence de contenu » (Éric Scherer, directeur Analyse stratégique et partenariats, AFP, 5 octobre 2007), partage de revenus entre le service d'infomédiation vidéo d'Orange et " un certain nombre de partenaires dont on sait qu'ils ont un petit peu plus de valeur que d'autres » (Laurent Eskenazi, responsable du projet 24/24 Actu Orange, 29 juin 2011) -, un accord collectif est finalement intervenu entre des entreprises médiatiques et un infomédiaire majeur devenus si interdépendants qu'ils n'avaient finalement plus d'autre choix que de s'entendre. En 2013, sous l'égide du président de la République François Hollande, un fonds financé par Google (Finp [Fonds pour l'innovation numérique dans la presse]) et cogéré par une association constituée de représentants des principaux newsmagazines et quotidiens nationaux français (Association de la presse d'information politique et générale) a été créé, se substituant d'ailleurs à une partie des aides publiques à la presse (Rebillard, 2018).

Au-delà de cet infléchissement en matière de politique publique, la mise en place de ce fonds a été critiquée par certains acteurs de la filière (en particulier les pure players par l'intermédiaire de leur syndicat professionnel Spiil) au motif que ses subsides étaient réservés aux médias les plus établis et conditionnés à l'utilisation des outils et des services de Google. En 2016, nous avons pu remarquer que ce type de critique demeurait chez un pure player :

«Dans ce que finance Google [...], il y a un biais très technologique et je ne suis pas très excité pour l'instant par les projets que je vois, je trouve que c'est très... c'est un peu artificiel. On met toujours de la data. Je trouve que journalistiquement, c'est pas forcément fascinant. [...] Donc voilà, il y a un peu une tension entre ce que c'est l'innovation dans la presse... Est-ce ce que finance Google ? Est-ce que c'est autre chose? " (Augustin 
Quelle « plateformisation » de l'information ? Collusion socioéconomique et dilution éditoriale entre les entreprises médiatiques et les infomédiaires de l'internet

Naepels, directeur financier et cofondateur, Les Jours, 28 novembre 2016)

Ce point de vue recueilli au cours de notre entretien, point de vue nécessairement situé d'un pure player français parmi d'autres, a depuis été corroboré par une inspection plus générale du fonds d'aide de Google aux médias à l'échelle européenne (DNI, Digital News Initiative Fund, 115 millions d'euros versés par Google de 2015 à 2017) : près de la moitié des projets financés (quatre sur dix) portait sur l'automatisation et le journalisme de données et pouvait, dans bien des cas, constituer une source d'externalités positives pour Google (Fanta, 2018).

Ce passage au peigne fin du fonds européen DNI a par ailleurs fait ressortir une tendance déjà entrevue quelques années auparavant avec le fonds français Finp, tendance consistant à principalement soutenir les médias les plus établis, issus de la presse écrite. Cette tendance générale a là aussi pu être illustrée dans le cadre de notre dernière vague d'enquête auprès d'un quotidien national, où la relation avec les infomédiaires semble vécue de façon beaucoup plus apaisée. Et, nouveauté en comparaison des années passées, la relation est désormais devenue interpersonnelle : ce ne sont plus des accords collectifs qui lient des firmes de bords opposés pour des périodes données, mais des individus qui, au sein de ces entreprises, échangent de façon directe et régulière. Ainsi, le responsable de la Snapteam du Monde, l'équipe de sept personnes chargée de créer les contenus du quotidien pour Snapchat, a une interlocutrice spécifique du côté de l'opérateur de plateforme:

" J'ai un contact éditorial avec qui je parle en particulier, qui est directrice des... enfin, qui est en charge de la plateforme Discover et qui est en charge de communiquer avec tous les éditeurs et de discuter avec eux. " (JeanGuillaume Santi, chef d'édition de la Snapteam, Le Monde, 24 novembre 2016)

Réciproquement, un dirigeant d'une start-up d'infomédiation indique lui aussi la généralisation et l'étroitesse de ces rapports avec des acteurs des médias. II fait ce constat à l'échelle de son entreprise émergente qui vise la vente d'article à l'unité, mais aussi à l'échelle de certains "géants " de l'Internet, 
Franck Rebillard et Nikos Smyrnaios

notamment ceux qui prospèrent grâce à la publicité :

«Et après, au niveau des prix, c'est pareil, on en discute avec les éditeurs. C'est eux qui mettent le prix, mais on discute quand même beaucoup avec eux. [...] [I] y a plein de gens qui sont partis de Apple News parce que finalement ils ont jamais partagé leurs données, parce qu'ils sont très stricts, vraiment c'est une boîte noire et ils sont pas très coopératifs, et nous c'est justement ce qu'on essaye de faire contre ça en donnant plein de données, en discutant, en secondant sur la stratégie, en les écoutant, voilà, pour pouvoir se différencier par rapport à ça. [...] On a en plus Google et Facebook qui ont des régies publicitaires et eux, qui ont tendance à discuter » (Pierre Tisserant, président et cofondateur OneMoreTab, président Articly, 8 novembre 2016)

Cette citation vient confirmer la collaboration croissante entre les personnels des entreprises médiatiques et ceux des infomédiaires (Nechushtai, 2018). Elle confirme aussi que le rapport de force du moment peut moduler le degré de cette collaboration: Google n'a déployé des efforts de relations véritablement humaines avec les journalistes ou les encadrants des rédactions qu'à partir du moment où son concurrent, Facebook, devenait inquiétant sur le marché de la publicité en ligne (Bell et Owen, 2017), et on perçoit ici que le petit outsider national qu'est Articly sur le marché du micropaiement doit davantage prospecter auprès des entreprises médiatiques que le leader mondial jusqu'ici incontesté qu'est Apple. Parallèlement, du côté des entreprises médiatiques, le pure player Les Jours s'avère beaucoup moins courtisé que le quotidien Le Monde, à l'audience et à la renommée bien plus grandes qui permettent à celui-ci d'être directement en contact avec Snapchat, selon une logique faisant écho à la différence de traitement réservée par les infomédiaires entre «larger publishers » et "smaller and local publications » aux États-Unis (Rashidian et al., 2018).

Les relations sont donc devenues plus étroites entre infomédiaires et entreprises médiatiques, ce qui pourrait constituer une caractéristique de la plateformisation. Cependant, on remarque que ces relations semblent être à géométrie variable en fonction de la position, dominante ou non, de chaque acteur. La collusion héritée de cette 
Quelle « plateformisation » de l'information ? Collusion socioéconomique et dilution éditoriale entre les entreprises médiatiques et les infomédiaires de l'internet

accentuation de l'infomédiation peut aussi se traduire par une collaboration entre une petite clique d'acteurs permettant d'en écarter d'autres.

\subsubsection{Imbrication commerciale}

La situation de coopétition caractérisant jusqu'ici l'infomédiation associait à une coopération entre acteurs fondée sur l'interdépendance de leurs services respectifs (mise à disposition de contenus par les entreprises médiatiques contre apport d'audience par les infomédiaires) une compétition commerciale sur de mêmes marchés, et principalement le marché de la publicité en ligne. Si le segment search du marché de la publicité en ligne a depuis le départ été préempté par les infomédiaires et demeure encore aujourd'hui l'apanage presque exclusif de Google (90\% des 2 milliards d'euros de ce segment en 2017 en France), le segment display (1,45 milliard d'euros) est, lui, disputé par différents types d'acteurs, dont les opérateurs de RSN (669 millions d'euros), les acteurs issus de la presse écrite (234 millions d'euros) et ceux issus de la télévision (126 millions d'euros) (estimations de Bearing Point pour le ministère de la Culture et le CSA, 2018).

Pendant plusieurs années, les entreprises médiatiques ont pu penser que, devancées sur le terrain du search auquel était associées une automatisation de la personnalisation publicitaire et une mesure instantanée de performance nécessitant d'avoir un savoir-faire technologique possédé par les infomédiaires, elles se rattraperaient sur le terrain du display en associant aux bannières publicitaires un contexte de qualité éditoriale adéquat pour les annonceurs (Ouakrat, Beuscart et Mellet, 2010). Comme nous le confiait ainsi un haut dirigeant du principal groupe de presse magazine français de l'époque :

" [La commercialisation de liens commandités par les annonceurs,] ce n'est pas notre métier. Notre métier va être plutôt d'investir dans la compréhension des besoins client. » (M. Julien Billot, Responsable de la direction numérique, Lagardère Active Media, 12 mars 2007)

Cependant, le segment display est loin d'être resté la chasse gardée des seules entreprises médiatiques. Ce segment s'est lui aussi teinté d'une forte coloration technologique ces dernières années, par le recours croissant à des procédés 
d'automatisation de la commercialisation publicitaire (publicité programmatique). Ainsi, selon les derniers chiffres disponibles à cette date, la commercialisation programmatique représentait près des deux tiers (62 \%) du segment display en France en 2017, augmentant ainsi de $41 \%$ par rapport à 2016, tandis que la commercialisation de gré à gré, représentant donc un tiers de ce segment (38\%), était en régression de $3 \%$ sur un an (Bearing Point, 2018).

Cet essor de la publicité programmatique pousse les entreprises médiatiques à doter leur activité de commercialisation publicitaire de compétences technologiques nouvelles, voire à mutualiser leurs moyens pour mettre en place de nouvelles régies communes et en partie automatisées (alliance Skyline entre les groupes Le Monde et Le Figaro, ou Gravity entre des entreprises médiatiques et des opérateurs de télécommunication/fournisseurs d'accès Internet). Or dans bien des cas également, les entreprises médiatiques vont passer par les régies publicitaires des infomédiaires pour commercialiser leurs espaces display, parachevant ainsi le processus de délégation de leur activité de vente de publicité en ligne, processus déjà entamé avec les liens commandités du search.

Sur ce plan, la plateformisation en tant qu'accentuation de l'infomédiation se traduit donc par une extension au display de la commercialisation des espaces publicitaires des entreprises médiatiques par les régies des infomédiaires, celles-ci s'étant multipliées en même temps que les plateformes développaient des services dédiés à l'information d'actualité, tels qu'Instant Articles (service de Facebook) ou Apple News. Nous avons pu relever ce phénomène de commercialisation publicitaire partagée (co-sale) dans le cas du service Discover de Snapchat pour Le Monde :

«Quand la régie publicité du Monde vend une campagne, ou quand la régie Snapchat vend une campagne, la publicité s'affiche comme une carte normale [carte Snapchat], mais toutes les trois cartes, donc 1, 2, 3, pub, $4,5,6$, pub, 7, 8, 9, pub [...]. Les deux régies sont capables de vendre des publicités aux annonceurs et derrière, il y a, quoi qu'il arrive, un partage de revenus entre Le Monde et Snapchat. " (Jean-Guillaume Santi, chef d'édition de la Snapteam, Le Monde, 24 novembre 2016) 
Quelle « plateformisation » de l'information ? Collusion socioéconomique et dilution éditoriale entre les entreprises médiatiques et les infomédiaires de l'internet

Ce partage de revenus se fait à parité entre Le Monde $(50 \%)$ et Snapchat $(50 \%)^{11}$. Dans le cas des services Instant Articles et Apple News, la commission prélevée par les régies des infomédiaires sur les publicités qu'elles commercialisent serait de $30 \%$. Dans tous les cas, il s'agit d'une perte de contrôle - au moins partielle - des entreprises médiatiques (et, en l'occurrence, des services marketing et de la régie publicitaire du Monde, phénomène confirmé par Salles [2018]) sur la commercialisation des publicités associées aux informations qu'elles ont pourtant produites. Ceci va donc bien au-delà de la domination qu'exerçaient déjà les infomédiaires dans la mise en visibilité des informations, et que continuent d'ailleurs de subir de plein fouet les entreprises médiatiques les plus en quête de notoriété :

"Alors nous, on n'est pas du tout à l'échelle où on peut contrarier quoi que ce soit. Mais, non, non, on essaye de se plier, afin de rentrer quand même, c'est important d'être référencé dans Google » (Augustin Naepels, directeur financier et cofondateur, Les Jours, 28 novembre 2016)

Si cette imbrication croissante dans la commercialisation publicitaire constitue un palier supplémentaire dans la domination des infomédiaires, l'emprise de ceux-ci sur la valorisation des données informatiques constitue peut-être un marqueur encore plus significatif de la plateformisation (Bullich, 2018). En apparence, une forme de collaboration entre les deux parties semble exister en la matière. Ainsi, l'infomédiaire que nous avons interrogé affirme-t-il :

"On donne accès aux données un peu classiques, des données assez classiques sur les usages, à la marque [marque $=$ média] $》$ (Pierre Tisserant, président et cofondateur OneMoreTab, président Articly, 8 novembre 2016).

À ce discours fait écho le propos de notre interlocuteur au Monde :

" On a accès à tout un ensemble de statistiques qui est hyper intéressant. On sait si les gens vont au bout de l'édition ou pas, on sait s'ils "swipent" pour lire les articles,

${ }^{11}$ Chiffres donnés par Laurence Bonicalzi-Bridier (présidente M Publicité - Groupe Le Monde) lors du colloque Marques, publicité et création audiovisuelle tenu à l'université Sorbonne Nouvelle - Paris 3 le 8 février 2018. 
combien de temps ils passent sur chaque article, on sait combien de gens viennent, des statistiques assez détaillées de behaviour au sein de l'application. " (JeanGuillaume Santi, chef d'édition de la Snapteam, Le Monde, 24 novembre 2016)

À y regarder de plus près, on se rend compte que seule une partie des données est rendue accessible aux entreprises médiatiques. II s'agit des données « comportementales », pour reprendre la terminologie marketing, renseignant sur les pratiques des internautes. En revanche, les données concernant l'identité et le profil sociodémographique de ces derniers, données les plus capitales et lucratives, ne semblent pas être dévoilées par les infomédiaires, ou alors de manière très parcellaire.

Dans le cas des données marketing comme dans le cas des publicités, la collaboration entre infomédiaires et entreprises médiatiques est donc plus poussée. Pour autant, le partage n'est pas équitable. II reste globalement à l'avantage des infomédiaires qui empiètent une fois de plus sur les platesbandes des entreprises médiatiques. Cependant, parmi celles$\mathrm{ci}$, on remarque, comme dans la section précédente, que toutes ne semblent pas logées à la même enseigne: certaines peuvent avoir leur mot à dire dans une négociation, certes inégale, quand d'autres sont complètement à l'écart de ces tractations.

\subsection{Dilution éditoriale}

Les indices de collusion socioéconomique identifiés précédemment ne sont pas sans lien avec un certain nombre de transformations de nature sémiodiscursive, tendant à fondre l'identité éditoriale de l'entreprise médiatique dans celle de la plateforme. L'identité éditoriale d'un média peut, pour les besoins de l'analyse, être décomposée en deux strates, celle de la ligne éditoriale et celle de l'énonciation éditoriale (Croissant Hare et Touboul, 2019), la première strate relevant plutôt de processus discursifs, quand la seconde renvoie plutôt à des dimensions technosémiotiques. Dans un tel cadre analytique, nous avançons l'argument d'une tendance à la dilution éditoriale se traduisant respectivement par une intervention grandissante et uniformisante des infomédiaires 
Quelle « plateformisation » de l'information ? Collusion socioéconomique et dilution éditoriale entre les entreprises médiatiques et les infomédiaires de l'internet

vis-à-vis des choix éditoriaux (choix des sujets à aborder, mode de traitement journalistique) des entreprises médiatiques et par un effacement progressif de l'énonciation médiatique au profit de sa prise en charge par l'infomédiaire (mise en forme adaptée aux attributs de chaque plateforme, pouvant passer par un hébergement physique des matériaux informatiques produits par l'entreprise médiatique) ${ }^{12}$.

Nous illustrerons ces évolutions à partir de captures d'écran d'informations produites par Le Monde et transitant par différentes plateformes. Là encore, nous sommes conscients du caractère très limité de cette observation empirique, ne pouvant révéler au mieux que des indices à propos d'un média particulier. Contraint, notre choix s'explique par la possibilité d'éclairer les traits sémiodiscursifs qui seront ainsi repérés à la lumière des considérations socioéconomiques glanées auprès de ce même média (entretien, et témoignages recueillis lors d'une conférence). En se portant sur Le Monde, l'analyse sémiodiscursive permettra par ailleurs de voir dans quelle mesure cette publication, bénéficiant d'un capital symbolique important dans le champ médiatique français et aujourd'hui particulièrement bien développée sur Internet (une des meilleures audiences en ligne), publication dont on a vu qu'elle pouvait en partie tirer son épingle du jeu de la collusion socioéconomique avec les infomédiaires, parvient à conserver ses attributs éditoriaux. Pour cela, l'accent est placé sur trois espaces d'infomédiation parmi les plus prisés par les médias de 2016 à 2018 (Apple, Facebook et Twitter cumulent la quasitotalité du volume des contenus identifiés dans l'étude de Rashidian et al. [2018], infra) ainsi que sur un espace un peu moins usité (Snapchat), mais qui a amené Le Monde, à cette occasion, à réviser drastiquement ses formats et son mode de couverture de l'actualité.

\footnotetext{
${ }^{12}$ Notre perspective se restreint à l'offre d'information en ligne. Cependant, l'analyse de ce phénomène de dilution éditoriale pourrait être profitablement prolongée jusqu'à la réception et à l'appropriation de l'information en ligne, où plusieurs études ont déjà pointé la difficulté des internautes à repérer l'entreprise médiatique énonciatrice d'origine, notamment dans les fils d'actualité de RSN où ils ne parviennent à identifier la source médiatique que dans $47 \%$ des cas (Kalogeropoulos et Newman, 2017 ) et se souviennent davantage de la personne ayant relayé l'information (Le Caroff, 2018).
} 


\subsubsection{Des contenus " natifs " émanant des plateformes}

Intitulée de façon volontairement provocante Platform Press (presse de plateforme), une étude s'est attelée récemment (Rashidian et al., 2018, mise à jour de Bell et Owen, 2017) à mesurer quantitativement le nombre d'informations diffusées par la voie de l'infomédiation aux États-Unis. Sur une période s'étalant d'août 2016 à mars 2018 a été scrutée la diffusion sur Apple News, Facebook, Instagram, li.st, LINE, LinkedIn, Messenger, Pinterest, Snapchat, Tumblr, Twitter, Vine et YouTube de deux chaînes de télévision (CNN, Fox News), de trois quotidiens à horizon national (The New York Times, The Wall Street Journal, The Washington Post), de trois autres quotidiens à l'horizon plus régional (Chicago Tribune, Los Angeles Times, New York Daily News) et de trois pure players (BuzzFeed, HuffPost, Vox). Cette diffusion s'est révélée intense, avec une moyenne hebdomadaire de 1800 contenus (de tous types: articles, photo, vidéo...) diffusés par média, et relativement stable sur l'ensemble de la période.

Dans le but d'affiner l'analyse, trois modes de diffusion des contenus ont été distingués entre contenus reliés (networked) " dont l'objectif premier est de ramener les internautes vers le site web du média ", contenus natifs (native), " hébergés nativement sur des plateformes tierces et pouvant être consultés gratuitement ", et contenus natifs payants (native paywalled) " diffusés sur des plateformes tierces et ne pouvant être consultés qu'après abonnement ${ }^{13}$ " (Rashidian et al., 2018, s. p.) (cette dernière formule est notamment employée par Apple News aux États-Unis). Les contenus diffusés sur un mode natif, que ce soit gratuitement (native) ou après paiement (native paywalled), ont représenté bon an mal an un peu moins de $50 \%$ de l'ensemble.

Notre propre observation, à propos du Monde, est de nature qualitative. Elle ne prétend donc pas, contrairement à la précédente, évaluer de façon chiffrée la proportion de contenus natifs qui ont été produits. Elle vise plutôt à identifier le degré d' " altération " subie par l'information, soit « la transformation active des discours et des textes médiatiques au fil [...] de leur

13 Traduction libre de: "whose primary purpose is to drive audiences back to a publisher's website ", " hosted natively on third-party platforms and could be viewed for free " et "made to third-party platforms that could only be viewed with a subscription ». 
Quelle « plateformisation » de l'information ? Collusion socioéconomique et dilution éditoriale entre les entreprises médiatiques et les infomédiaires de l'internet

circulation entre différents espaces sociaux » (Jeanneret, 2014, p. 10). Une altération sémiodiscursive d'ampleur au cours de la période récente serait alors considérée comme un indice du passage par un espace social relevant d'une infomédiation exacerbée s'apparentant à la plateformisation.

De ce point de vue, la version Snapchat du Monde est plutôt saisissante. La copie d'écran reproduite ci-dessous (Figure 1) montre des partis-pris éditoriaux assez radicaux pour ce journal quotidien que l'on qualifie couramment de " sérieux » ou « de référence $"$.
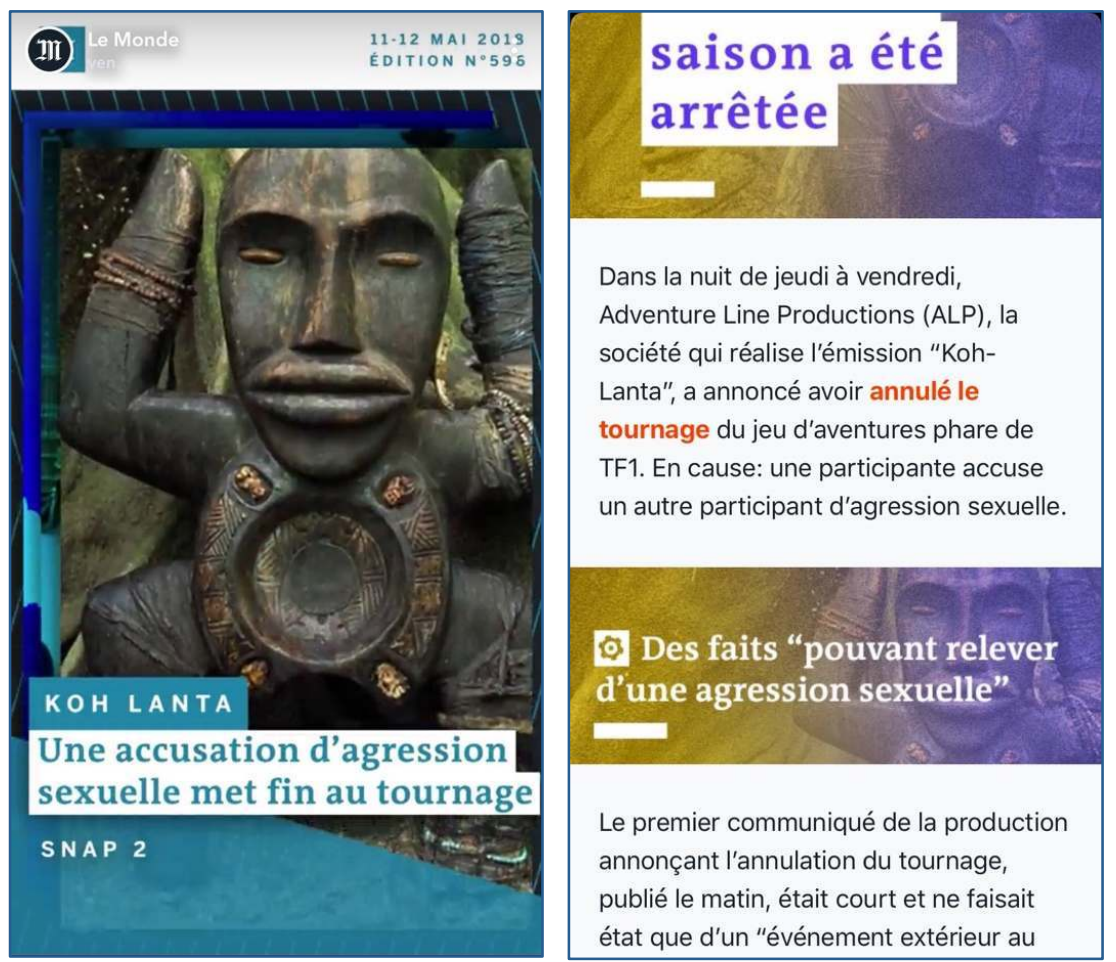

Figure 1. Captures d'écran de Snapchat Discover Le Monde (mai 2018)

D'une part, sur le plan discursif, le sujet - agression sexuelle d'une participante à un jeu télévisé - certes se rapporte à l'actualité du printemps 2018 marquée par la sensibilisation aux violences faites aux femmes à la suite des dénonciations concernant le producteur de cinéma Harvey Weinstein et à la suite du mouvement \#MeToo, mais cette thématique est ici 
mise en avant par le biais d'une émission de télévision - Koh Lanta - qui n'a habituellement pas les faveurs du Monde. D'autre part et peut-être surtout, sur le plan sémiotique, la place laissée à l'iconographie est immense : images et couleurs sont ici inhabituelles pour une publication qui, malgré le lancement de son site web et de son édition magazine du dimanche ces dernières décennies, s'est longtemps caractérisée par une sobriété qui interdisait notamment le recours à la photographie.

Cette double "révolution » éditoriale peut être rattachée à des facteurs socioéconomiques. Lors de notre entretien, le responsable de l'édition de la Snapteam a rappelé à plusieurs reprises la volonté du Monde de toucher une population plus jeune grâce à Snapchat, à laquelle répond très probablement l'évocation d'une émission de télévision particulièrement populaire auprès de cette catégorie d'âge. Le recours à une mise en forme chatoyante peut aussi s'expliquer par cette stratégie de conquête marketing d'un nouveau " segment » de lecteurs. II s'inscrit sans doute plus fondamentalement encore dans l'interpénétration entre Le Monde et Snapchat, matérialisée concrètement par l'hébergement informatique des fichiers de l'éditeur sur la plateforme, et activée plus quotidiennement par l'adaptation aux formats requis :

« Donc on arrive avec un set de contraintes en fait qui sont décidées par Snapchat. [...] Dans les TopSnaps, il y a des petites vidéos de une qui ne peuvent pas durer plus de 10 secondes. Donc si la vidéo, elle dure 11 secondes, elle n'est pas acceptée pas le CMS en fait. [...] Pour Le Monde, il y a vraiment un côté très expérimental, pour nous, de ne plus maîtriser les tuyaux. " (Jean-Guillaume Santi, chef d'édition de la Snapteam, Le Monde, 24 novembre 2016)

Le Monde doit donc se résoudre à accepter les conditions ergonomiques de Snapchat s'il veut profiter de ce canal pour rajeunir son lectorat. Dans les propos qui suivent, le responsable de la Snapteam du Monde révèle son attention aux conseils prodigués par son interlocutrice chez Snapchat, selon une forme d'interaction assez proche du stories pitching observée entre infomédiaires et éditeurs aux États-Unis (Rashidian et al., 2018), tout en insistant sur la conservation d'un libre-arbitre éditorial pour le journal. L'intérêt de s'entendre semble ainsi mutuellement bien compris par l'un des médias les 
Quelle « plateformisation » de l'information ? Collusion socioéconomique et dilution éditoriale entre les entreprises médiatiques et les infomédiaires de l'internet

plus puissants sur le territoire national comme par l'un des infomédiaires les plus en pointe au moment de notre enquête ${ }^{14}$ :

"Quand on discute avec Snapchat, évidemment ils nous donnent des conseils, [...] on n'a pas la prétention d'arriver sur une plateforme et de dire on a tout compris de cette plateforme alors qu'on y est à peine arrivés. Donc ils nous disent que, je ne sais pas, pour que les gens s'avancent un peu plus dans votre édition, il y a cette astuce de faire comme-ci ou de faire comme ça. Et du coup, des fois, on essaye. [...] Ce qui est important c'est que sur le niveau du contenu éditorial on ait toujours, quoi qu'il arrive, le final cut. " (Jean-Guillaume Santi, chef d'édition de la Snapteam, Le Monde, 24 novembre 2016)

La plateforme Snapchat est singulière en ce sens qu'elle a connu le succès grâce à l'utilisation de l'image. On peut dès lors comprendre que les producteurs d'information incités à rejoindre Snapchat doivent se fondre dans ce format très " icono-centré » et, par là même, déposer les lourds fichiers informatiques attenants sur le serveur de la plateforme pour en faciliter l'affichage. Ces arguments techniques d'ergonomie ont également été employés par les autres plateformes pour proposer leurs services de contenus "natifs", tels que Facebook Instant Articles ou Apple News. Au moment de notre observation, au printemps 2018, seul celui-ci concernait Le Monde (Figure 2).

\footnotetext{
${ }^{14}$ Depuis moins en vogue qu'Instagram, Snapchat a néanmoins vu la composante informative de son usage continuer à croître : en 2018, cet usage concerne $6 \%$ des Français et même $13 \%$ des personnes âgées de 18 à 24 ans (RISJ, 2018).
} 

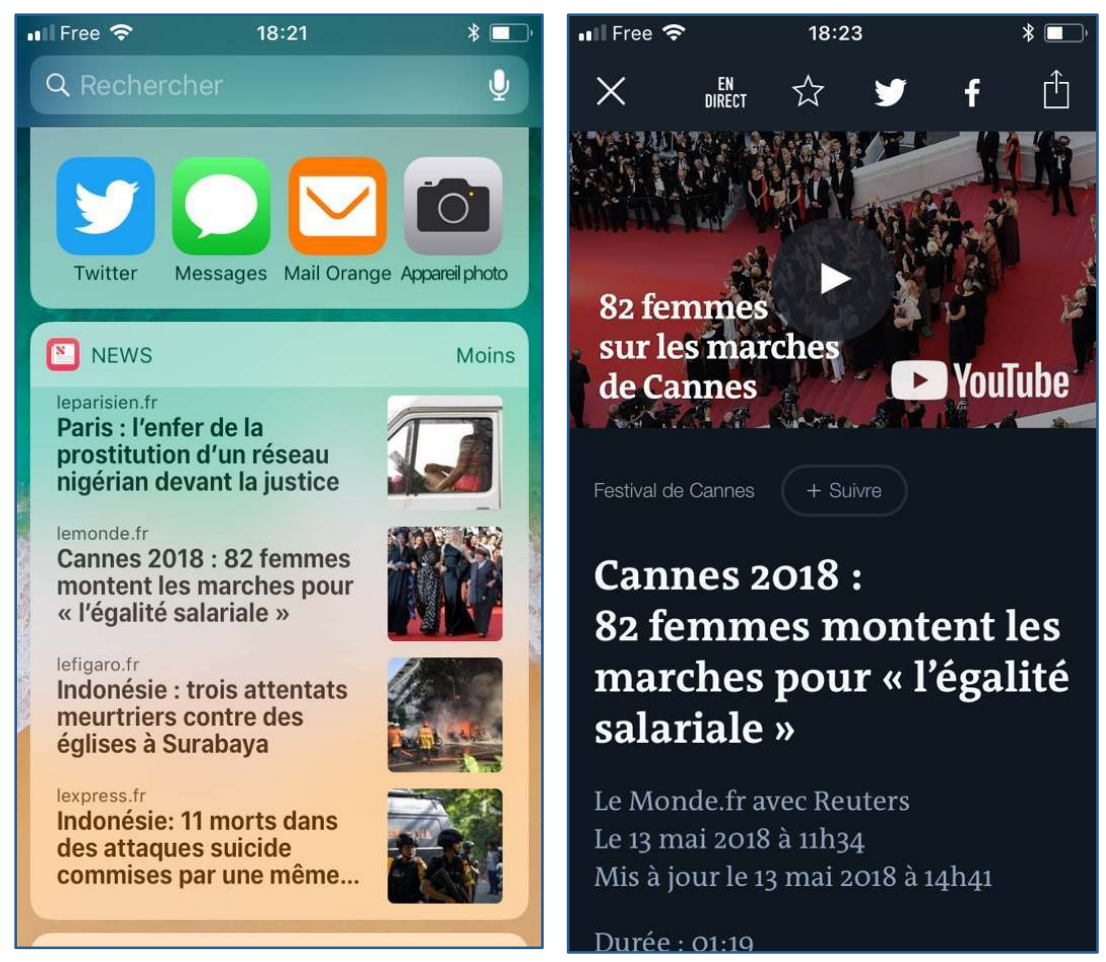

Figure 2. Captures d'écran du widget d'Apple News et du Monde App (mai 2018)

Nous avons ici volontairement sélectionné un sujet qui se rapportait également à l'actualité découlant de l'affaire Weinstein. En comparaison de ce qui a pu être observé sur Snapchat, nous remarquons tout d'abord, sur le plan discursif, que le sujet est davantage en phase avec la ligne éditoriale traditionnelle du Monde, puisque le journal accorde depuis de longues années une grande importance au Festival de Cannes, à la différence de Koh Lanta.

Sur le plan sémiotique par ailleurs, la mise en forme est assurément plus sobre que sur Snapchat et s'écarte ainsi moins des standards du Monde. Elle témoigne cependant d'un effacement énonciatif de l'entreprise médiatique au profit d'une forme de neutralité sémiotique qui profite à l'infomédiaire. Le logo du Monde a ainsi complètement disparu sur le widget Apple News, et tous les noms des entreprises médiatiques présentes sont rendus à une même typographie. Ceci ne constitue pas complètement une nouveauté si l'on songe, par exemple, au fait que Google News adoptait une même 
Quelle « plateformisation » de l'information ? Collusion socioéconomique et dilution éditoriale entre les entreprises médiatiques et les infomédiaires de l'internet

uniformisation typographique voilà déjà plus d'une décennie (Figure 3, archive de Google Actualités en 2008, au moment de la même période du Festival de Cannes afin de faciliter la comparaison), sauf que plusieurs éléments nouveaux apparaissent ici. D'une part, le widget Apple News ne renvoie pas forcément au site web du Monde (comme c'était le cas en 2008 par hyperlien, Figure 4), mais à l'application Le Monde issue de l'Apple Store : l'internaute reste donc confiné dans le périmètre informatique de l'infomédiaire, qui peut alors prendre possession de ses données personnelles si précieuses sur le plan socioéconomique. D'autre part, l'interface Le Monde App elle-même offre la possibilité d'autres data channels, cette fois par une vidéo YouTube incrustée (cf. Figure 2), témoignant d'une omniprésence de la double logique de plateformisation (Helmond, 2015), logique bien moins poussée il y a dix ans de cela, où l'icône " Partager " paraissait bien seule et discrète en comparaison (Figure 4).

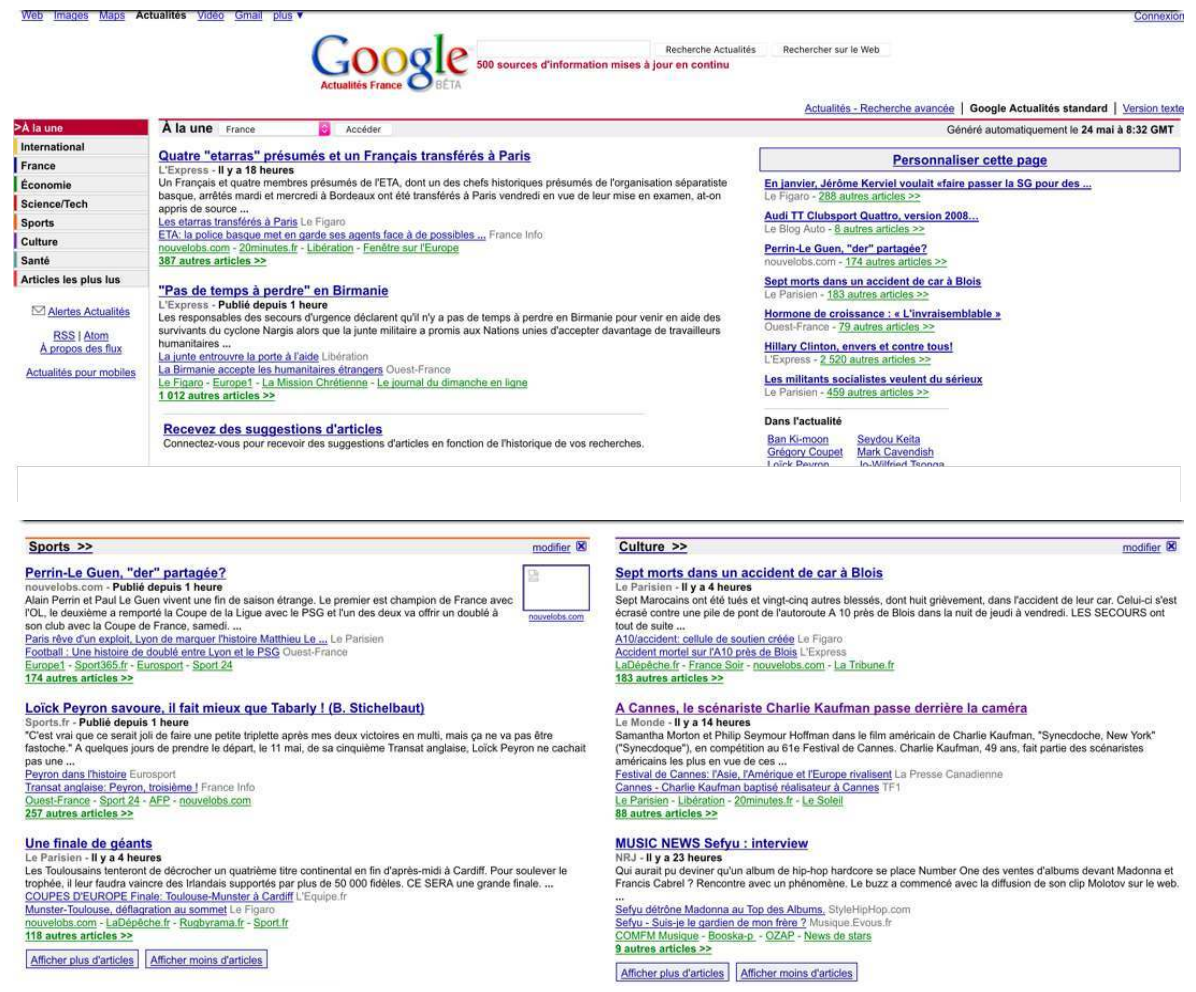

Figure 3. Captures d'écran de Google Actualités (mai 2008) 


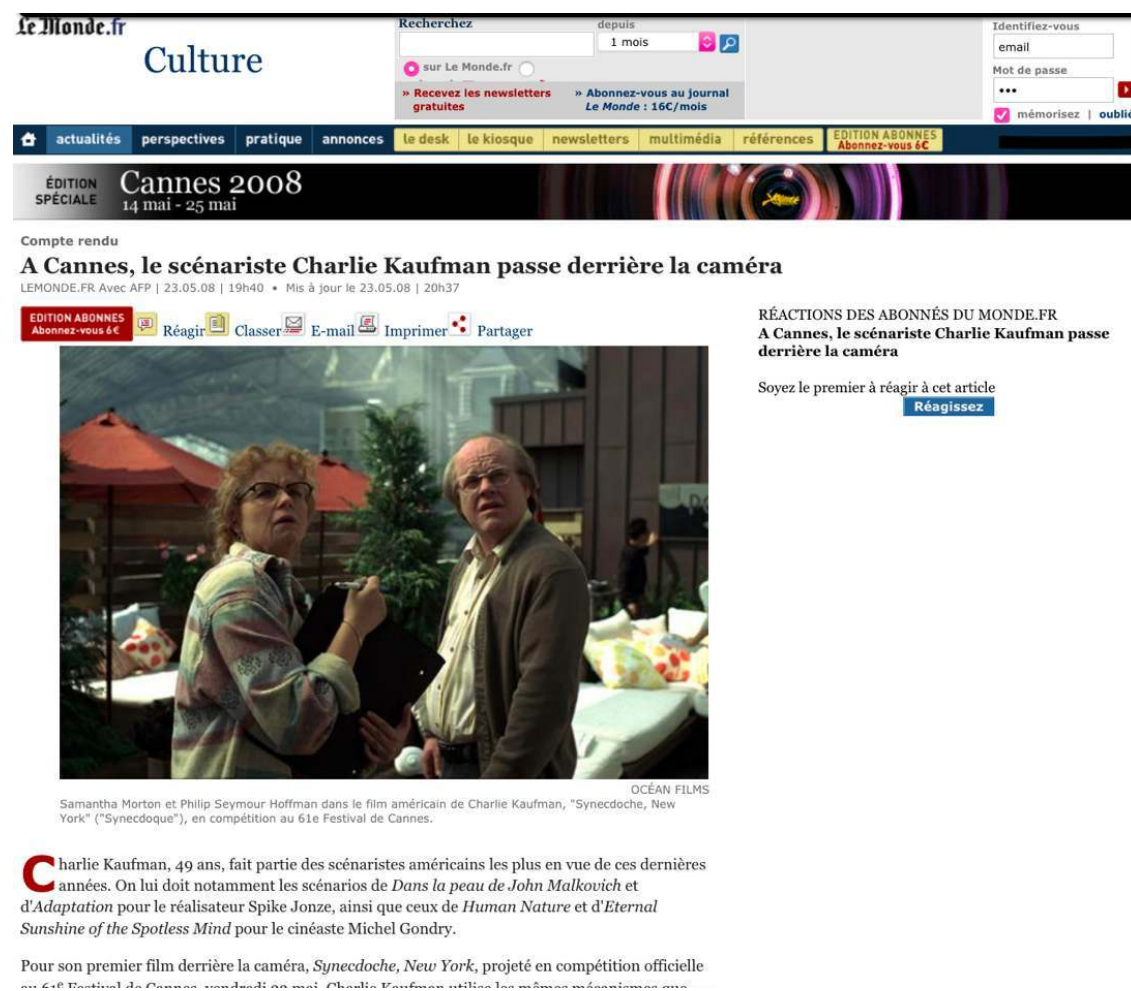

Figure 4. Capture d'écran du site web Le Monde (mai 2008)

\subsubsection{Une hégémonie du moule éditorial des plateformes}

Les exemples de la section précédente ont montré que les infomédiaires pouvaient aller jusqu'à héberger les informations produites par les entreprises médiatiques, ou en tout cas à les maintenir dans leur périmètre informatique, ce qui constituerait un indice supplémentaire de plateformisation. Sans aller toujours jusqu'à cette mainmise concrète sur les fichiers, l'accentuation de l'emprise des infomédiaires sur le plan éditorial se concrétise de façon plus diffuse par l'orientation architextuelle (Jeanneret et Souchier, 2005) donnée au mode de présentation des informations sur les plateformes.

Une illustration de cette tendance est à nouveau fournie par Le Monde, cette fois dans sa relation à Facebook. Dans un premier temps, Le Monde a pu recevoir des incitations monétaires de Facebook, comme d'autres entreprises et groupes bien établis dans le paysage médiatique national, en échange du recours aux contenus natifs lancés par la firme 
Quelle « plateformisation » de l'information ? Collusion socioéconomique et dilution éditoriale entre les entreprises médiatiques et les infomédiaires de l'internet

californienne et, en particulier, aux vidéos Facebook Live (aides versées par Facebook à différents médias d'un montant de 100000 à 200000 euros par mois, et financement de studios de production dédiés dans certaines rédactions, comme celles de LCl, RTL ou Europe 1, d'après Becquet [2017]). Après avoir ainsi contribué - indirectement - à assurer la promotion de ces contenus natifs de Facebook et à en faire des formats standards de l'information en ligne adoptés par d'autres médias moins puissants (et non aidés par Facebook), Le Monde a, comme d'autres médias dominants français ou états-uniens qui avaient eux aussi momentanément touché de l'argent pour cela, soudainement ou progressivement abandonné cette voie (Rashidian et al., 2018). En l'espèce, d'après les dires d'une des dirigeantes du groupe ${ }^{15}$, Le Monde ne souhaitait pas être totalement dépendant des plateformes et se sentait assez " costaud " pour s'en passer. Ceci renforce une nouvelle fois l'idée d'une inégalité entre les médias vis-à-vis de leur pouvoir de négociation - Le Monde possède des ressources supplémentaires à d'autres - avec les infomédiaires, tout comme la variabilité du pouvoir réciproque de ceux-ci Facebook peut conjoncturellement perdre de son attractivité au profit de Snapchat.

Quoi qu'il en soit, au moment de notre observation (printemps 2018), les fichiers informatiques du Monde ne sont donc pas hébergés d'emblée sur les serveurs de Facebook et l'internaute est renvoyé vers le site mobile.lemonde.fr (Figure 5).

\footnotetext{
${ }^{15}$ Propos de Laurence Bonicalzi-Bridier (présidente M Publicité - Groupe Le Monde), 8 février 2018.
} 


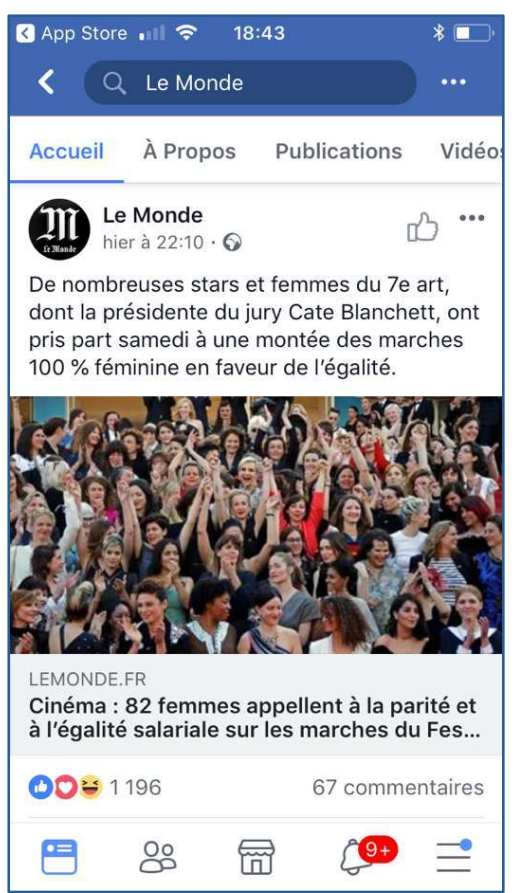

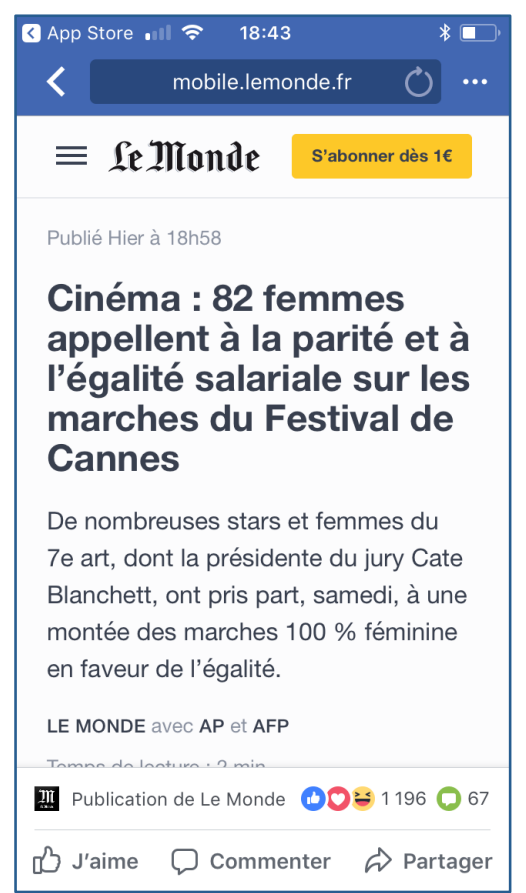

Figure 5. Captures d'écran de Facebook App et du site web mobile Le Monde (mai 2018)

On peut alors constater, sur le plan sémiodiscursif, que pour ce même sujet de la protestation cannoise en faveur de l'égalité salariale entre femmes et hommes dans le cinéma, les attributs éditoriaux du Monde sont plus manifestes sur Facebook (cf. Figure 5) qu'ils ne l'étaient sur Apple News (cf. Figure 2) : le logo de l'entreprise médiatique est de retour et un chapô (chapeau introductif) développe l'information sans s'arrêter au seul titre.

Pour autant, la forme-modèle de présentation du Monde sur Facebook (cf. Figure 5) ne fait pas référence à la lecture prointensive, lecture en profondeur et exigeant de la concentration, qui pouvait être attachée au quotidien dit "sérieux", mais plutôt à une lecture pro-extensive :

La police sans empattement pour le texte courant est censée favoriser la lecture rapide à l'écran. Un haut niveau de tabularité de la page-écran, une structuration des articles mettant en avant les "informations » principales du texte, des encadrés synthétiques, mais aussi le marquage fort des hyperliens incitant 
Quelle « plateformisation » de l'information ? Collusion socioéconomique et dilution éditoriale entre les entreprises médiatiques et les infomédiaires de l'internet

préférentiellement à une lecture fragmentée peuvent être considérés comme des formes-modèles pro-extensives. (Saemmer, 2015, s. p.)

Ces formes-modèles pro-extensives, moins perceptibles sur le site web du Monde il y a une dizaine d'années (cf., au sein de la Figure 4, la police avec empattement ou la présence moins intempestive d'hyperliens dans le texte), semblent de fait moins héritées de la tradition journalistique du Monde que d'une rationalisation informatique croissante qui pourrait être l'empreinte des infomédiaires.

Un autre indice allant en ce sens est fourni par le mode de présentation des informations du Monde sur Twitter (Figure 6) tant il ressemble à celui affiché sur Facebook (cf. Figure 5). Certes le discours est un peu différent : les titres ne reprennent pas exactement les mêmes termes et les prises de vue des photographies, bien que portant toutes deux sur les marches du Palais du Festival de Cannes, varient. Cependant, la mise en forme d'ensemble demeure: logo, titre et chapô dans une police sans empattement, photographie, encadré, densité d'hyperliens. 

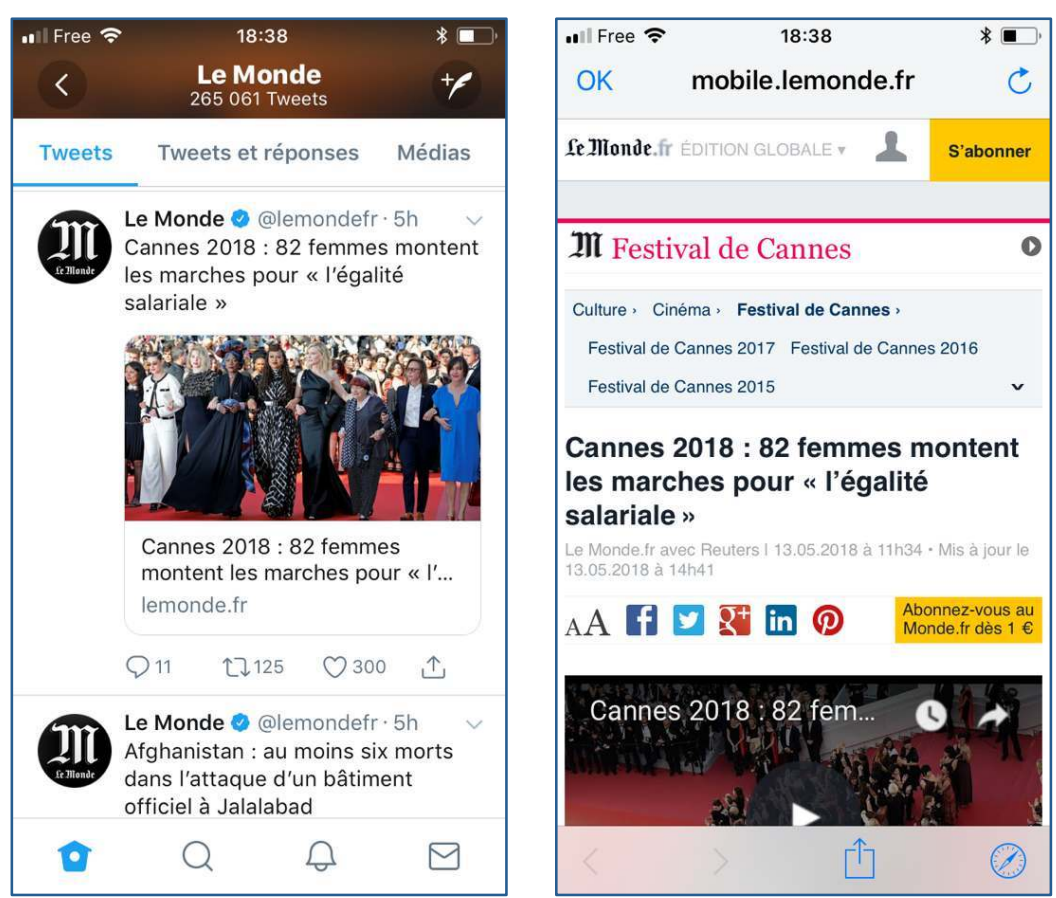

Figure 6. Captures d'écran de Twitter App et du site web mobile Le Monde (mai 2018)

Poussée jusqu'au niveau sociotechnique de la programmation informatique, l'analyse sémiodiscursive peut expliquer de telles parentés formelles. Comme cela a pu être analysé pour plusieurs autres " cartes Twitter » de ce type, les développeurs des pages mobiles du Monde ont dû inclure dans le code source des spécifications instruisant sa rééditorialisation sur ce RSN comme sur d'autres espaces d'infomédiation (Goyet, 2017). Concrètement, du côté de l'entreprise médiatique, les développeurs ont la possibilité de choisir quels titres ou quelles illustrations s'afficheront sur telle ou telle interface d'infomédiaire, par l'intermédiaire de balises de programmation. Néanmoins, l'architecture dans laquelle viendront se loger ces différents modules est rigide. Elle est élaborée par les infomédiaires et les entreprises médiatiques n'ont pas prise sur elle: les premiers sont en position de stratège et les secondes renvoyées à une position de tacticien, selon la distinction certalienne.

Ainsi, même lorsque les entreprises médiatiques ne vont pas jusqu'à transférer leurs fichiers informatiques aux infomédiaires, 
Quelle « plateformisation » de l'information ? Collusion socioéconomique et dilution éditoriale entre les entreprises médiatiques et les infomédiaires de l'internet

elles en viennent tout de même à se couler dans leur moule éditorial, en passant par tout un éventail allant de la primoéditorialisation à la méta-éditorialisation (Sonet, 2019). Alors que l'expression "désosser le journal » employée par un de nos enquêtés (Jean-Charles Falloux, responsable production web, Les Échos, 14 mars 2007) désignait à l'époque du XML triomphant la simple opération de démodularisation du quotidien papier pour faciliter l'intégration des articles aux différentes rubriques du site web du journal, c'est depuis une industrialisation et une automatisation du réassemblage éditorial vers une multitude de plateformes qui s'est développée. Une telle généralisation de la dilution éditoriale ajoute une pierre supplémentaire à l'édifice de la plateformisation dont on avait déjà vu la poussée socioéconomique : " II y a là une anticipation de la circulation qui passe par des pratiques d'écritures - le code informatique -, pratiques qui se déploient dans un contexte économique et industriel de valorisation de la circulation. " (Goyet, 2017, p. 87.)

\section{Conclusion}

Caractérisées dans une première phase de coopétition par des rapports faits à la fois de coopération inévitable et de concurrence à distance, fondées sur une modularisation en micro-unités informatives et débouchant sur un dualisme éditorial, les relations entre infomédiaires de l'Internet et entreprises médiatiques feraient ainsi place à une situation contemporaine de collution (collusion socioéconomique et dilution éditoriale mêlées). Une partie des entreprises médiatiques semble se plier à une collusion avec les infomédiaires pour leurs activités de production journalistique et de gestion commerciale de la publicité ainsi qu'à une dilution de leurs choix et de leurs formats éditoriaux vis-à-vis de ceux des principaux infomédiaires.

Plus précisément, parmi les entreprises médiatiques, toutes ne sont pas assujetties de la même manière aux infomédiaires : dans les cas étudiés, nous avons pu, par exemple, percevoir qu'en comparaison d'un nouvel entrant, le média qui occupe la position la plus haute dans le champ est en mesure de peu ou prou négocier les termes de sa relation à un infomédiaire ou de 
délaisser les services promus par un autre. Ceci incite à penser que, réciproquement, du côté des infomédiaires, certains peuvent avoir moins le vent en poupe après avoir été conjoncturellement dominants.

Un double mouvement semble ainsi se dessiner, ce dont tente de rendre compte de façon synthétique le Tableau 1. Le mouvement majeur paraît bien être celui d'une intégration verticale accrue entre les deux catégories d'acteurs, entreprises médiatiques et infomédiaires, mais un autre mouvement de concurrence horizontale au sein de chacune de ces catégories d'acteurs tend à le traverser. Pour reprendre la distinction certalienne déjà évoquée, on pourrait ainsi dire que, si les médias s'apparentent majoritairement à des tacticiens liés à des infomédiaires stratèges, ces deux ensembles ne sont pas homogènes et encore moins unis, à tel point que des alliances entre fractions dominantes de chaque groupe peuvent voir le jour pour mieux évincer la concurrence et s'imposer. 
Quelle « plateformisation » de l'information ? Collusion socioéconomique et dilution éditoriale entre les entreprises médiatiques et les infomédiaires de l'internet

Tableau 1. Synthèse - La plateformisation comme accentuation de l'infomédiation : tendances principales

\begin{tabular}{|c|c|c|c|c|c|c|}
\hline Phases & \multicolumn{3}{|c|}{ Tnfomédiaíion } & \multicolumn{3}{|c|}{ Plateformis ation (phase d'accentuation de linfomédiation) } \\
\hline Dimensions & $\begin{array}{l}\text { Situation } \\
\text { globale }\end{array}$ & $\begin{array}{l}\text { Niveau vertical: } \\
\text { infomédiaires } / \\
\text { producteurs } \\
\text { d'information } \\
\end{array}$ & $\begin{array}{l}\text { Niveau horizontal: } \\
\text { intra producteurs } \\
\text { d'information }\end{array}$ & $\begin{array}{l}\text { Evolufion de } \\
\text { la situation } \\
\text { globale }\end{array}$ & $\begin{array}{l}\text { Indices d'évolution au } \\
\text { niveau vertical : } \\
\text { infomédiaires i } \\
\text { producteurs } \\
\text { d'information } \\
\end{array}$ & $\begin{array}{l}\text { Indices d'évolution au } \\
\text { niveau horizontal: inta } \\
\text { producteurs } \\
\text { d'information }\end{array}$ \\
\hline Sociologique & & $\begin{array}{l}\text { Tnterdépendance peu } \\
\text { formalisée : } \\
\text { - Souvent absence de } \\
\text { relations } \\
\text { - Parfois relations } \\
\text { collectives entre } \\
\text { infomédiaire et } \\
\text { groupement de } \\
\text { producteurs } \\
\text { - Rarement accords } \\
\text { bilatéraux entre } \\
\text { infomédiaire et } \\
\text { producteur particulier }\end{array}$ & $\begin{array}{l}\text { - Relations collectives } \\
\text { menées par } \\
\text { groupements de } \\
\text { producteurs majeurs } \\
\text { - Accords particuliers } \\
\text { avec des producteurs } \\
\text { majeurs } \\
\text { - Absence de relations } \\
\text { pour les autres }\end{array}$ & & $\begin{array}{l}\text { Intégration renforcée se } \\
\text { concrétisant par des } \\
\text { relations } \\
\text { interpersonnelles }\end{array}$ & $\begin{array}{l}\text { - Interlocuteurs } \\
\text { désignés chez les } \\
\text { infomédiaires et chez } \\
\text { certains producteurs } \\
\text { majeurs } \\
\text { - Absence de relations } \\
\text { pour les autres } \\
\text { producteurs } \\
\text { d'information }\end{array}$ \\
\hline Economique & Coopétition & $\begin{array}{l}\text { Concurrence sur le } \\
\text { marché de la publicité } \\
\text { en ligne : } \\
\text { - Dévelopement de la } \\
\text { publicitié personnnalisée } \\
\text { search (liens } \\
\text { sponsorisés) } \\
\text { commercialisée par les } \\
\text { régies des } \\
\text { infomédiaires } \\
\text { - Publicité display } \\
\text { restant maitisée par } \\
\text { les producteurs } \\
\text { d'information } \\
\text { (Concurrence sur le } \\
\text { marché de laventeen } \\
\text { ligne: développement } \\
\text { du micro-piement, } \\
\text { parallelement àla } \\
\text { modalité plus classique } \\
\text { d'abonnement) }\end{array}$ & $\begin{array}{l}\text { - Possibilité pour les } \\
\text { producteurs majeurs } \\
\text { de commercialiser } \\
\text { directementh leurs } \\
\text { publicitís voire leurs } \\
\text { abonnements grâaeeà } \\
\text { leur notoriétée et image } \\
\text { de marque. } \\
\text { - Fins d'inenentaire } \\
\text { search pour les } \\
\text { producteurs mineurs }\end{array}$ & $\begin{array}{l}\text { Collusion } \\
\text { socio- } \\
\text { économique }\end{array}$ & $\begin{array}{l}\text { Concurrenceétendue } \\
\text { au disslay avec le } \\
\text { développement de la } \\
\text { publicitée } \\
\text { programmatique } \\
\text { Multiplication des } \\
\text { formules de } \\
\text { commercialisation } \\
\text { partagée entre } \\
\text { infomédiaires et } \\
\text { producteurs (cos-sale) } \\
\text { pour la publicité et aussi } \\
\text { pour la vente } \\
\text { d'information, amplifíés } \\
\text { par l'accès aux données } \\
\text { des utilisateurs }\end{array}$ & $\begin{array}{l}\text { - Tentatives dalliance } \\
\text { entre producteurs } \\
\text { majeurs dans la } \\
\text { publicité } \\
\text { programmatique } \\
\text { - Dépendance accrue } \\
\text { aux infomédiaires pour } \\
\text { les autres producteurs } \\
\text { d'information }\end{array}$ \\
\hline $\begin{array}{l}\text { Techno- } \\
\text { sémiotique }\end{array}$ & & $\begin{array}{l}\text { Modularisation } \\
\text { éditoriale: biens- } \\
\text { systèmes médiatiques } \\
\text { désassemblés en } \\
\text { micro-unités } \\
\text { informatives pouvant } \\
\text { être reliées voire } \\
\text { réassemblées. }\end{array}$ & $\begin{array}{l}\text { Différences de } \\
\text { visibilité des micro- } \\
\text { unités } \\
\text { informatives selon } \\
\text { leurs producteurs } \\
\text { d'origine: } \\
\text { capacités de } \\
\text { référencement } \\
\text { supérieures pour les } \\
\text { producteurs majeurs } \\
\text { (et plus rarement } \\
\text { accords avec les } \\
\text { infomédiaires) }\end{array}$ & & $\begin{array}{l}\text { Accentuation du } \\
\text { réassemblage méta- } \\
\text { éditorial par les } \\
\text { infomédiaires: } \\
\text { - Hébergement des } \\
\text { fichiers et contrôle des } \\
\text { data channels (applis, } \\
\text { widgets, contenus } \\
\text { natifs) } \\
\text { - Emprise de la forme } \\
\text { modèle des plateformes }\end{array}$ & $\begin{array}{l}\text { - Faveurs accordées } \\
\text { aux producteurs } \\
\text { majeurs } \\
\text { (subventionnement de } \\
\text { contenus natiss, } \\
\text { sélection dans des } \\
\text { perimetres } \\
\text { d'infomédiation de plus } \\
\text { en plus réduits) } \\
\text { - Situation subie par les } \\
\text { autres producteurs } \\
\text { d'information }\end{array}$ \\
\hline Discursif & $\begin{array}{l}\text { et dualisme } \\
\text { éditorial }\end{array}$ & $\begin{array}{l}\text { Dualisme entre } \\
\text { similaritée editoriale } \\
\text { (principalement tirée } \\
\text { par les infomédiaires et } \\
\text { les producteurs } \\
\text { d'information } \\
\text { traditionnels et } \\
\text { originalité ed idtoriale } \\
\text { (principalement activée } \\
\text { par les producteurs } \\
\text { d'information natifis de } \\
\text { l'intermet) }\end{array}$ & $\begin{array}{l}\text { - Suivisme editoria } \\
\text { explicable par le } \\
\text { recours des } \\
\text { producteurs } \\
\text { traditionnels aux outils } \\
\text { et procédés des } \\
\text { infomédiaires } \\
\text { (analytics, metrics, } \\
\text { trends) } \\
\text { - Information } \\
\text { alternative } \\
\text { volontairementen } \\
\text { marge de ces } \\
\text { pratiques et axée sur } \\
\text { le libre-arbitre éditorial } \\
\end{array}$ & $\begin{array}{l}\text { Dilution } \\
\text { éditoriale }\end{array}$ & $\begin{array}{l}\text { Possible nivellement de } \\
\text { la ligne éditoriale des } \\
\text { producteurs } \\
\text { d'information les plus au } \\
\text { contact des } \\
\text { infomédiaires. }\end{array}$ & $\begin{array}{l}\text { Intervention humaine } \\
\text { des infomédiaires } \\
\text { auprès des producteus } \\
\text { d'information } \\
\text { traditionnels touchant } \\
\text { aux processus de } \\
\text { création (training } \\
\text { sessions, stories } \\
\text { pitching) }\end{array}$ \\
\hline
\end{tabular}

Assurément, une telle situation est beaucoup plus complexe que ce que l'appellation courante de plateformisation laisse supposer de monolithique. Si elle semble bien consister en une accentuation générale de la prégnance de l'infomédiation en matière d'information en ligne, ce serait néanmoins pour prendre des directions et des atours fort variés. Pour autant, nous resterons prudents à ce stade vis-à-vis d'une telle conclusion, pour au moins deux raisons. La première tient au caractère réduit de l'étude empirique présentée ici, sur lequel nous avons déjà insisté. Elle peut fournir quelques indices 
nourrissant notre hypothèse générale, mais ne peut prétendre à la traiter complètement. La seconde raison est liée à l'inscription incertaine de ces mouvements dans la longue durée. Un infléchissement vers la plateformisation ressenti aujourd'hui, aussi polymorphe soit-il, ne signifie aucunement sa pérennisation sur un terme plus étendu. Surtout, il ne doit pas faire oublier que d'autres configurations sociotechniques (Rebillard, 2007) pourront émerger ou se renforcer.

Notre conclusion est donc forcément provisoire et située. En termes d'économie politique de la communication, elle peut néanmoins d'ores et déjà alerter, car se dessinent, au travers d'une intégration socioéconomique et éditoriale accentuée, des asymétries elles-mêmes croissantes. Or celles-ci se produisent dans un domaine sensible pour la vie en société, celui de l'information. À cette aune, si la situation de collution venait à se confirmer et à perdurer (voire à prendre des formes encore plus poussées, comme les initiatives de coproduction de programmes audiovisuels d'information entre Facebook et des chaînes de télévision pourraient en augurer, cf. PignardCheynel [2018]), une régulation publique plus adaptée devrait se faire jour vis-à-vis d'infomédiaires qui se défendent encore aujourd'hui de toute responsabilité éditoriale malgré de nombreux faits contraires pourtant susceptibles de les soumettre aux obligations citoyennes régissant les entreprises médiatiques (Napoli et Caplan, 2017).

Dans le même temps, il faudrait aussi veiller à ce que cette collution ne se double pas d'ententes ciblées entre grands infomédiaires et grandes entreprises médiatiques, à la manière des dérives déjà maintes fois observées dans la "grande distribution » entre fournisseurs de biens établis et réseaux oligopolistiques (Allain et Chambolle, 2003). Car à la différence des produits de consommation courante, l'information a besoin de diversité, en provenance de médias de toutes envergures, et non des seuls médias mainstream que le recours croissant à l'infomédiation semble pourtant favoriser (Nechushtai et Lewis, 2018) au détriment d'acteurs de plus petite taille, mais qui représentent bien souvent des foyers de pluralisme. 
Quelle « plateformisation » de l'information ? Collusion socioéconomique et dilution éditoriale entre les entreprises médiatiques et les infomédiaires de l'internet

\section{Références}

Águila-Obra (Del), A. R., Padilla-Meléndez, A. et SerarolsTarrés, C. (2007). Value creation and new intermediaries on Internet: An exploratory analysis of the online news industry and the web content aggregators. International Journal of Information Management, 27(3), 187-199.

Allain, M.-L. et Chambolle, C. (2003). Économie de la distribution. Paris, France : La Découverte.

AT-Internet (2015). Les lecteurs de la presse online optent de plus en plus pour le smartphone. Repéré à https://www.atinternet.com/ressources/etudes/

Bearing Point (2018). Médias et publicité en ligne. Transfert de valeur et nouvelles pratiques. Étude pour le ministère de la Culture et le Conseil supérieur de l'audiovisuel. Repéré à http://www.culture.gouv.fr/Thematiques/Audiovisuel/Rapports -etudes/Etude-sur-les-Medias-et-la-publicite-en-lignetransfert-de-valeur-et-nouvelles-pratiques2

Becquet, N. (2017). Facebook a versé des millions aux médias français : la stratégie "VIP-VRP » et ses effets. European Journalism Observatory. Repéré à https://fr.ejo.ch/economiemedias/facebook-remuneration-medias-francais-live-video

Bell, E. et Owen (2017, 29 mars). The Platform Press. How Silicon Valley Reengineered Journalism. Tow Center for Digital Journalism. Repéré à https://www.cjr.org/tow_center reports/platform-press-how-silicon-valley-reengineeredjournalism.php

Bouquillion, P., Miège, B. et Mœglin, P. (2013). L'industrialisation des biens symboliques: les industries créatives en regard des industries culturelles. Grenoble, France : Presses universitaires de Genoble.

Bullich, V. (2018). La "plateformisation » de la formation. Distances et médiations des savoirs, (21). Repéré à http://journals.openedition.org/dms/2096

Bustamante, E. (2004). Cultural industries in the digital age: Some provisional conclusions. Media, Culture \& Society, 26(6), 803-820.

Croissant, V., Hare, I. et Touboul, A. (2019, janvier). Éditorialiser l'actualité pour les réseaux sociaux numériques : 
entre opportunisme et innovation journalistique des nouveaux acteurs de l'information sur le web. Communication présentée lors du Colloque international Journalisme et plateformes, Toulouse.

Fanta, A. (2018). Le nouveau mécène : comment Google étend son emprise sur le journalisme européen. European Journalism Observatory. Repéré à https://fr.ejo.ch/innovation-et-numerique/google-emprisemedias-mecene

Goyet, S. (2017). Outils d'écriture du web et industrie du texte. Du code informatique comme pratique lettrée. Réseaux, (206), 61-94.

Helmond, A. (2015). The platformization of the web: Making web data platform ready. Social Media + Society, 1(2), 1-11.

Hesmondhalgh, D. (2013). The Cultural Industries. Londres, Royaume-Uni : Sage Publications.

Jeanneret, Y. (2014). Critique de la trivialité. Les médiations de la communication, enjeux de pouvoir. Paris, France : Éditions Non Standard.

Jeanneret, Y. et Souchier, E. (2005). L'énonciation éditoriale dans les écrits d'écran. Communication et langages, (145), 3-15.

Just, N. et Latzer, M. (2017). Governance by algorithms: Reality construction by algorithmic selection on the internet. Media, Culture \& Society, 39(2), 238-258.

Kalogeropoulos, A. et Newman, N. (2017). "I Saw the News on Facebook". Brand Attribution when Accessing News from Distributed Environments. Reuters Institute for the Study of Journalism.

Kalogeropoulos, A. et Nielsen, R. K. (2017). Investing in Online Video News. Journalism Studies, 19(15). doi: https://doi.org/10.1080/1461670X.2017.1331709

Le Caroff, C. (2018). Facebook, média de masse : un poids à relativiser. La Revue des Médias. Repéré à

https://larevuedesmedias.ina.fr/facebook-media-de-masse-unpoids-relativiser 
Quelle « plateformisation » de l'information ? Collusion socioéconomique et dilution éditoriale entre les entreprises médiatiques et les infomédiaires de l'internet

Lewis, S. C. et Westlund, O. (2015). Actors, Actants, Audiences, and Activities in Cross-Media News Work: A Matrix and a Research Agenda. Digital Journalism, 3(1), 1937.

Miège, B., Pajon, P. et Salaün, J.-M. (1986). L'industrialisation de l'audiovisuel. Des programmes pour les nouveaux médias. Paris, France : Aubier-Montaigne.

Mœglin, P. (2007). Des modèles socioéconomiques en mutation. Dans P. Bouquillion et Y. Combès (dir.), Les industries de la culture et de la communication en mutation (p. 151-162). Paris, France : L'Harmattan.

Napoli, P. M. (2014). Automated Media: An Institutional Theory Perspective on Algorithmic Media Production and Consumption. Communication Theory, (24), 340-360.

Napoli, P. M. et Caplan, R. (2017). Why media companies insist they're not media companies, why they're wrong, and why it matters. First Monday, 22(5). doi: http://dx.doi.org/10.5210/ fm.v22i15.7051

Nechushtai, E. (2018). Could digital platforms capture the media through infrastructure?. Journalism, 19(8), 1043-1058.

Nechushtai, E. et Lewis, S. C. (2018). What kind of news gatekeepers do we want machines to be? Filter bubbles, fragmentation, and the normative dimensions of algorithmic recommendations. Computers in Human Behaviour, (90), 298-307. doi: https://doi.org/10.1016/j.chb.2018.07.043

Nielsen, R. K. et Ganter, S. A. (2018). Dealing with Digital Intermediaries: A Case Study of the Relations Between Publishers and Platforms. New Media \& Society, 20(4), 1600-1617.

Ouakrat, A., Beuscart J.-S. et Mellet K. (2010). Les régies publicitaires des sites de la presse quotidienne. Réseaux, (160-161), 133-161.

Pew Research Center (Pew). (2016). State of the News Media 2016. Repéré à https://assets. pewresearch.org/wpcontent/uploads/sites/13/2016/06/30143308/state-of-thenews-media-report-2016-final.pdf 
Pew Research Center (Pew). (2018a). News Use Across Social Media Platforms 2018. Repéré à https://www.journalism.org/ 2018/09/10/news-use-across-social-media-platforms-2018/

Pew Research Center (Pew). (2018b). Appendix D - Topline Questionnaire. Western Europeans Under 30 View News Media Less Positively, Rely More on Digital Platforms Than Older Adults. Repéré à https://www.journalism.org/2018/10 130/western-europeans-under-30-view-news-media-lesspositively-rely-more-on-digital-platforms-than-older-adults/

Pignard-Cheynel, N. (2018). Facebook et les médias: une relation diaboliquement complexe. La Revue des Médias. Repéré à https://larevuedesmedias.ina.fr/facebook-et-lesmedias-une-liaison-diaboliquement-complexe

Plantin, J.-C., Lagoze, C., Edwards, P. N. et Sandvig, C. (2018). Infrastructure Studies Meet Platform Studies in the Age of Google and Facebook. New Media \& Society, 20(1). doi: https://doi.org/10.1177/1461444816661553

Poell, T., Nieborg, D., Brooke, E. D., Prey, R. et Cunningham, S. (2017). The Platformization of Cultural Production. Selected Papers of \#AolR2017 - The 18 ${ }^{\text {th }}$ Annual Conference of Internet Researchers. Repéré à http://www.gamespace.nl/content/AolR2017-Platformizationof-Cultural-Production.pdf

Rashidian, N., Brown, P. et Hansen, E., avec Bell, E., Albright, J., et Hartstone, A. (2018, 14 juin). Friend and Foe: The Platform Press at the Heart of Journalism. Columbia Journalism Review. Repéré à https://www.cjr.org/tow_center reports/the-platform-press-at-the-heart-of-journalism.php/

Rebillard, F. (2012). Modèles socioéconomiques du journalisme en ligne et possibilités d'une information diversifiée. Les enjeux de l'information et de la communication. https://lesenjeux.univ-grenoble-alpes.fr/2011supplement/Rebillard/Rebillard.pdf

Rebillard, F. (2007). Le web 2.0 en perspective. Une analyse socioéconomique de l'internet. Paris, France : L'Harmattan.

Rebillard, F. (2018). Le financement de la presse et de l'information en ligne en France. Évolutions et enjeux. Dans L. Ballarini, S. Costantini, M. Kaiser, J. Matthews et V. Rouzé (dir.), Financement participatif. Les nouveaux territoires du 
Quelle « plateformisation » de l'information ? Collusion socioéconomique et dilution éditoriale entre les entreprises médiatiques et les infomédiaires de l'internet

capitalisme. Nancy, France : PUN-Éditions universitaires de Lorraine.

Rebillard, F. (sous presse). Médias analogiques, médias numériques: des industries socio-symboliques. Dans B. Lafon (dir.), L'analyse des médias. Grenoble, France: Presses universitaires de Grenoble.

Rebillard, F., George, E., Goyette-Côté M.-O. et Smyrnaios, N. (2011). Enjeux socioéconomiques de la diffusion d'informations d'actualité sur l'internet. Les relations entre éditeurs de presse et industries de la communication. Rapport de recherche pour le ministère de la Culture et de la Communication.

Rebillard, F. et Smyrnaios, N. (2011). Entre coopération et concurrence : les relations entre infomédiaires et éditeurs de contenus d'actualité. Concurrences, 8(3), 7-18.

RISJ - Reuters Institute for the Study of Journalism (2018). Digital News Report 2018. Repéré à https://reutersinstitute.politics.ox.ac.uk/

Saemmer, A. (2015). Préfigurations du lecteur dans la presse en ligne. Une analyse sémio-rhétorique de la pratique des hyperliens par les webjournalistes. Communication, 33(2). doi: $10.4000 /$ communication. 6030

Salles, C. (2018). Les compétences de modération dans le journal Le Monde: réquisition, redéfinition et redistribution. Le Temps des médias, (31), 48-61.

Siapera, E. (2013). Platform infomediation and journalism. Culture Machine, (14), 1-28.

Smyrnaios, N. (2015). Google and the Algorithmic Informediation of News. Media Fields, (10), 1-10.

Smyrnaios, N. (2017). Les Gafam contre l'internet. Une économie politique du numérique. Bry-sur-Marne, France: Ina Éditions.

Smyrnaios, N. et Rebillard, F. (article en cours d'évaluation). How infomediation platforms took over the news. A longitudinal perspective.

Sonet, V. (2019, janvier). Apple News et Google Actualités : ces widgets révélateurs des stratégies de méta-éditorialisation 
des plateformes. Communication présentée lors du Colloque international Journalisme et plateformes, Toulouse.

Tambini, D. et Labo, S. (2016). Digital intermediaries in the UK: Implications for news plurality. Info, 18(4), 33-58.

Tandoc, E. C. Jr et Maitra, J. (2018). News organizations' use of Native Videos on Facebook: Tweaking the journalistic field one algorithm change at a time. New Media \& Society, 20(5), 1679-1696.

Winseck, D. (2016). Reconstructing the Political Economy of Communication for the Digital Media Age. The Political Economy of Communication, 4(2), 73-114. 